\title{
An Efficient Class of WENO Schemes with Adaptive Order
}

\author{
By \\ Dinshaw S. Balsara ${ }^{1}$, Sudip Garain ${ }^{1}$ and Chi-Wang Shu ${ }^{2}$ \\ 1 (dbalsara@nd.edu, sgarain@nd.edu) Physics Department, Univ. of Notre Dame \\ 2(hu@dam.brown.edu) Division of Applied Mathematics, Brown University
}

\begin{abstract}
Finite difference WENO schemes have established themselves as very worthy performers for entire classes of applications that involve hyperbolic conservation laws. In this paper we report on two major advances that make finite difference WENO schemes more efficient.
\end{abstract}

The first advance consists of realizing that WENO schemes require us to carry out stencil operations very efficiently. In this paper we show that the reconstructed polynomials for any one-dimensional stencil can be expressed most efficiently and economically in Legendre polynomials. By using Legendre basis, we show that the reconstruction polynomials and their corresponding smoothness indicators can be written very compactly. The smoothness indicators are written as a sum of perfect squares. Since this is a computationally expensive step, the efficiency of finite difference WENO schemes is enhanced by the innovation which is reported here.

The second advance consists of realizing that one can make a non-linear hybridization between a large, centered, very high accuracy stencil and a lower order WENO scheme that is nevertheless very stable and capable of capturing physically meaningful extrema. This yields a class of adaptive order WENO schemes, which we call WENO-AO (for adaptive order). Thus we arrive at a $\operatorname{WENO}-\operatorname{AO}(5,3)$ scheme that is at best fifth order accurate by virtue of its centered stencil with five zones and at worst third order accurate by virtue of being non-linearly hybridized with an $r=3$ CWENO scheme. The process can be extended to arrive at a WENO-AO(7,3) scheme that is at best seventh order accurate by virtue of its centered stencil with seven zones and at worst third order 
accurate. We then recursively combine the above two schemes to arrive at a WENO$\operatorname{AO}(7,5,3)$ scheme which can achieve seventh order accuracy when that is possible; graciously drop down to fifth order accuracy when that is the best one can do; and also operate stably with an $r=3 \mathrm{CWENO}$ scheme when that is the only thing that one can do. Schemes with ninth order of accuracy are also presented.

Several accuracy tests and several stringent test problems are presented to demonstrate that the method works very well.

\section{I) Introduction}

Ever since the early papers by Harten et al. [14] and Shu \& Osher [29], [30] there has been a great deal of interest in Essentially Non-Oscillatory (ENO) schemes that can obtain the solution to hyperbolic conservation laws with better than second order accuracy in the vicinity of smooth flows. ENO schemes come in two flavors. The original ENO schemes by Harten et al. [14] were based on finite volume discretizations, where the conserved variable is reconstructed using the smoothest stencil possible. A more efficient variant of ENO schemes by Shu \& Osher [29], [30] relies on finite difference discretizations, where upwinding is applied directly to the fluxes on a dimension-bydimension basis. When the physical problem develops discontinuities, the ENO schemes rely on non-linear hybridization and upwinding to stabilize the solution. The early ENO schemes suffered from their own pathologies. As a result, weighted ENO (WENO) schemes were invented to overcome those deficiencies (Liu, Osher \& Chan [23], Jiang \& Shu [19]). The methods were extended to eleventh order by Balsara \& Shu [1] and more recently to seventeenth order by Gerolymos, Sénéchal \& Vallet [13]. A formulation of WENO that preserves accuracy at critical points was presented in Henrick, Aslam \& Powers [15], Borges et al. [3] and Castro et al. [4]. For a comprehensive review of WENO schemes, see Shu [31]. In this paper we focus on finite difference WENO schemes. 
WENO methods achieve their high order by analyzing all the stencils, including the highly one-sided ones, that enable one to reconstruct the solution within a zone with $r^{\text {th }}$ order polynomials. It was realized that when the flow is smooth, all possible stencils provide an equally good reconstruction. Thus in smooth regions of flow, a convex combination of all possible stencils may be taken to yield $(2 r-1)^{\text {th }}$ order of accuracy. The linear weights that are ascribed to all the different stencils are then called optimal linear weights because they help optimize the accuracy of the scheme. The one-sided stencils can potentially provide upwinding and stability in the vicinity of shocks, with the result that the reconstruction strategy should emphasize the stencil/s that yield the smoothest interpolation in such regions.

It is easiest to understand how WENO schemes achieve stability at discontinuities if one briefly considers total variation diminishing (TVD) schemes. In TVD methods stability is achieved in the vicinity of discontinuities by picking a one-sided slope with the help of a solution-dependent, non-linear limiter. Picking a left-biased slope and a right-biased slope is equivalent to picking a left- and right-biased stencils, each of which has two zones. The limiter provides non-linear hybridization by examining the one-sided slopes/stencils and picking out the slope with the smaller absolute value. In WENO schemes one first identifies all the possible stencils that cover a zone of interest with a suitably high order reconstruction polynomial. The stencils can be one-sided or centered. When the flow is smooth, we wish to pick out the centered stencil or a linear combination of all the stencils so as to optimize order of accuracy. When the flow has discontinuities, we wish to pick out the stencil with the smoothest possible solution. The choice of stencil is, therefore, made solution-dependent leading to a non-linearly hybridized scheme even when the governing equation may perhaps be linear. The non-linear stabilization is achieved by assigning a solution-dependent smoothness indicator to each of the stencils. At a technical level, the smoothness indicator for a given stencil is the sum of the squares of all the derivatives that are present in the reconstruction polynomial associated with that stencil. The weights assigned to the different stencils are such that if all stencils have the same smoothness indicators (i.e. the stencils are equally good interpolants in the vicinity of smooth flow) then the weights tend to the optimal linear weights. It is by this device 
that the accuracy of conventional WENO schemes is improved. However, when one or a few stencils have substantially smaller smoothness indicators than the rest (i.e., a sub-set of stencils are much better interpolants in the vicinity of shocks) then the smoothest stencils carry the highest weight with the non-smooth stencils carrying a vanishingly small weight. It is by this device that the WENO schemes achieve their non-linear hybridization. Recently, Zhu and Qiu [37] have non-linearly hybridized a fifth order reconstruction polynomial with a Van Albada-like limiter to arrive at a scheme that is fifth order for smooth flow and second order TVD at discontinuities. In situations where the Van Albada limiter is invoked even a little, extrema will be clipped. It is, therefore, desirable to do better.

The finite difference WENO schemes described above have shown themselves to be versatile performers. Numerical studies of turbulence require careful attention to accuracy and phase errors, as shown in the compact schemes of Lele [21]; see also Tam and Webb [35]. Compact-WENO schemes have, therefore, been designed to handle shocks and simultaneously increase the phase accuracy by Pirozzoli [27], Shen and Yang [32], Deng and Zhang [7]. See also Hu et al. [18], Martin et al. [25] and Johnsen et al. [20] for further information on low dispersion schemes for turbulence. The finite difference WENO methods have also been adapted to handle complex geometry by $\mathrm{Hu}$ and Shu [16] and Liu and Zhang [24]. We see, therefore, that it is very desirable to improve the efficiency and accuracy of this class of schemes. This is done in the next two paragraphs.

It is easy to see that rather large stencils need to be analyzed in the course of carrying out WENO reconstruction. Because of the size of the stencils being analyzed, it helps to have the most efficient strategies for evaluating the reconstructed polynomials as well as the smoothness indicators. The expressions provided in Jiang \& Shu [19], Balsara \& Shu [1] and Gerolymos, Sénéchal \& Vallet [13] are not the most compact ones that can be obtained. In particular, Balsara et al. [2] realized that analyzing the problem in a basis set formed by Legendre polynomials yields a mathematically equivalent formulation that is computationally more efficient. While the expressions from Balsara et al. [2] were 
shown to be useful for finite volume WENO schemes up to fourth order, an extension of the same expressions for finite difference WENO schemes with increasingly high order of accuracy is extremely valuable. The first goal of this paper is to provide such a formulation up to $r=9$. The resulting expressions for the reconstructing polynomials are very compact and suitable for implementation in numerical codes that use up to seventeenth order accurate finite difference WENO. The expressions for the smoothness indicators have a very compact form but they also have the added advantage that they can be written as a sum of perfect squares, thus making their positive nature evident.

While the classical finite difference WENO schemes emphasize high accuracy, there are other WENO-type schemes which emphasize stability. The central WENO (CWENO) schemes (Levy, Puppo \& Russo [22], Cravero \& Semplice [6], Semplice, Coco \& Russo [28]), which have also been extended to unstructured meshes by Friedrichs [12], Käser and Iske [17] and Dumbser and Käser [10], fall in this category of WENO schemes. Such schemes emphasize the central, and most stable, stencil over and above all the other stencils. Thus for smooth flow, the method always tends to the most stable central stencil. For non-smooth flow, the smoothness indicators permit the reconstruction to pick out the smoothest one-sided stencil. Experience has shown that the $r=3$ CWENO is extremely stable, rivaling the stability of TVD schemes. All the CWENO schemes also preserve physical extrema if such extrema exist in the flow. This enables us to realize the limitation of the WENO-ZQ scheme of Zhu and Qiu [37] which nonlinearly hybridized a central stencil with an extrema-clipping Van Albada limiter. We avoid the clipping of extrema in this paper by achieving a non-linear hybridization between a central fifth order reconstruction polynomial along with the $r=3$ CWENO reconstruction. We call such finite difference schemes WENO-AO for adaptive order. The resulting adaptive order WENO-AO scheme, which we denote as $\operatorname{WENO}-\mathrm{AO}(5,3)$, is fifth order accurate where it is possible and at least third order accurate in situations where the fifth order accuracy might produce unphysical extrema. Because of the nonlinear hybridization with the $r=3$ CWENO reconstruction, it will also be stable while simultaneously avoiding the clipping of physical extrema. Accuracy testing shows that our WENO-AO $(5,3)$ scheme can be almost half an order of magnitude more accurate 
than the WENO-ZQ scheme for certain test problems. We also demonstrate a suitable non-linear hybridization between a central seventh order stencil and the three CWENO stencils with $r=3$. This yields a WENO-AO $(7,3)$ reconstruction strategy with an adaptive order that can range between $7^{\text {th }}$ order (at best) and $3^{\text {rd }}$ order (at worst). We then realize that we can recursively make a suitable non-linear hybridization between the WENO$\mathrm{AO}(7,3)$ reconstruction strategy and the WENO-AO(5,3) reconstruction strategy. The resulting WENO-AO(7,5,3) reconstruction gives us a 100\% stable strategy of switching from a seventh order scheme to a fifth order scheme and further switching from fifth order to third order. We also show that WENO-AO(9,3) and WENO-AO(9,5,3) schemes can be constructed. The second goal of this paper is to catalogue the finite difference WENO-AO schemes of different orders. Please note that the nomenclature emphasizes the spatial accuracy of the finite difference WENO schemes since all such schemes achieve their temporal accuracy via Runge-Kutta time update methods.

Section II presents the formulation of WENO reconstruction in a space of Legendre basis functions. Section III catalogues efficient WENO-AO schemes with adaptive order. Section IV presents some accuracy results. Section V applies the schemes to several stringent test problems. Section VI presents conclusions.

\section{II) Formulation of WENO Reconstruction in Legendre Basis}

The Legendre polynomials, suitably modified for the domain $[-1 / 2,1 / 2]$, are given by: 


$$
\begin{aligned}
& \mathrm{L}_{0}(x)=1 ; \mathrm{L}_{1}(x)=x ; \mathrm{L}_{2}(x)=x^{2}-\frac{1}{12} ; \mathrm{L}_{3}(x)=x^{3}-\frac{3}{20} x ; \\
& \mathrm{L}_{4}(x)=x^{4}-\frac{3}{14} x^{2}+\frac{3}{560} ; \mathrm{L}_{5}(x)=x^{5}-\frac{5}{18} x^{3}+\frac{5}{336} x ; \\
& \mathrm{L}_{6}(x)=x^{6}-\frac{15}{44} x^{4}+\frac{5}{176} x^{2}-\frac{5}{14784} ; \\
& \mathrm{L}_{7}(x)=x^{7}-\frac{21}{52} x^{5}+\frac{105}{2288} x^{3}-\frac{35}{27456} x ; \\
& \mathrm{L}_{8}(x)=x^{8}-\frac{7}{15} x^{6}+\frac{7}{104} x^{4}-\frac{7}{2288} x^{2}+\frac{7}{329472}
\end{aligned}
$$

In the remaining Sub-sections we catalogue the WENO reconstruction in the basis space provided by these Legendre polynomials for $r=3$ to $r=9$. In this paper we are only interested in addressing finite difference WENO, so we restrict our attention to onedimensional stencils. The $r=3$ to 5 cases have been catalogued in Balsara et al. [2] and are repeated here because we wish to show that the emerging pattern in the smoothness indicators is very general and can be used for designing WENO schemes with even larger values of " $r$ ". The $r=6$ to 9 cases are new.

Potentially, the $r=9$ case can lead to the design of classical finite difference WENO schemes with $17^{\text {th }}$ order spatial accuracy, i.e. an accuracy that is comparable to the most accurate finite difference WENO schemes in Gerolymos et al. [13]. The design of such very high order WENO schemes will be greatly helped by the fact that our expressions for the smoothness indicators in this paper are extremely compact. It is not our intention to design $17^{\text {th }}$ order WENO schemes in this paper. However, it is our intention to show that a very general procedure has been found for designing WENO schemes with adaptive order. In other words, the WENO-AO (for adaptive order) schemes can automatically pick out stencils of suitably high order. The goal of the present section is to catalogue the compact expressions for the reconstruction and the smoothness indicators. The detailed description of WENO-AO will be provided in the next section.

\section{II.1) $r=3$ WENO Reconstruction}


We focus on the reconstruction problem in a zone labeled by a subscript " 0 ". Consider the neighboring zone-averaged variables $\left\{\mathrm{u}_{-2}, \mathrm{u}_{-1}, \mathrm{u}_{0}, \mathrm{u}_{1}, \mathrm{u}_{2}\right\}$. A third order reconstruction over the zone labeled " 0 " can be carried out by using the left-biased stencil $S_{1}^{r 3}$, the centered stencil $S_{2}^{r 3}$ and the right-biased stencil $S_{3}^{r 3}$ that rely on the variables $\left\{\mathrm{u}_{-2}, \mathrm{u}_{-1}, \mathrm{u}_{0}\right\},\left\{\mathrm{u}_{-1}, \mathrm{u}_{0}, \mathrm{u}_{1}\right\}$ and $\left\{\mathrm{u}_{0}, \mathrm{u}_{1}, \mathrm{u}_{2}\right\}$ respectively. In this paper we label our stencils with a superscript that denotes the $r^{\text {th }}$ order of the polynomial and a subscript that denotes the stencil for that $r$-value. Specification of these two numbers always allows us to specify a precise reconstruction polynomial with a specified smoothness indicator. The $i^{\text {th }}$ reconstructed polynomial corresponding to stencil $S_{i}^{r 3}$ is then expressed as

$$
\mathrm{P}_{i}^{r 3}(x)=\mathrm{u}_{0}+\mathrm{u}_{x} \mathrm{~L}_{1}(x)+\mathrm{u}_{x 2} \mathrm{~L}_{2}(x)
$$

The stencil $S_{1}^{r 3}$ gives

$$
\mathrm{u}_{x}=-2 \mathrm{u}_{-1}+\mathrm{u}_{-2} / 2+3 \mathrm{u}_{0} / 2, \mathrm{u}_{x 2}=\left(\mathrm{u}_{-2}-2 \mathrm{u}_{-1}+\mathrm{u}_{0}\right) / 2
$$

The stencil $\mathrm{S}_{2}^{r 3}$ gives

$\mathrm{u}_{x}=\left(\mathrm{u}_{1}-\mathrm{u}_{-1}\right) / 2 \quad, \mathrm{u}_{x 2}=\left(\mathrm{u}_{-1}-2 \mathrm{u}_{0}+\mathrm{u}_{1}\right) / 2$

The stencil $\mathrm{S}_{3}^{r 3}$ gives

$\mathrm{u}_{x}=-3 \mathrm{u}_{0} / 2+2 \mathrm{u}_{1}-\mathrm{u}_{2} / 2, \mathrm{u}_{x 2}=\left(\mathrm{u}_{0}-2 \mathrm{u}_{1}+\mathrm{u}_{2}\right) / 2$

The smoothness indicator for each of the three stencils can then be written in a very compact form which is a sum of two squares as 
$\beta^{r 3}=\left(\mathrm{u}_{x}\right)^{2}+\frac{13}{3}\left(\mathrm{u}_{x 2}\right)^{2}$

For each stencil $\mathrm{S}_{i}^{r 3}$, we can add a subscript " $i$ " to the smoothness indicator in eqn. (2.6) to denote that it corresponds to a specific stencil.

\section{II.2) $r=4$ WENO Reconstruction}

In considering the $r=4$ WENO reconstruction problem in a zone labeled by a subscript " $0 "$, we focus on the neighboring zone-averaged variables $\left\{\mathrm{u}_{-3}, \mathrm{u}_{-2}, \mathrm{u}_{-1}, \mathrm{u}_{0}, \mathrm{u}_{1}, \mathrm{u}_{2}, \mathrm{u}_{3}\right\}$. The fourth order reconstruction can be carried out by using four stencils $\mathrm{S}_{1}^{r 4}, \mathrm{~S}_{2}^{r 4}, \mathrm{~S}_{3}^{r 4}$ and $\mathrm{S}_{4}^{r 4}$ that rely on the variables $\left\{\mathrm{u}_{-3}, \mathrm{u}_{-2}, \mathrm{u}_{-1}, \mathrm{u}_{0}\right\}$, $\left\{\mathrm{u}_{-2}, \mathrm{u}_{-1}, \mathrm{u}_{0}, \mathrm{u}_{1}\right\} \quad\left\{\mathrm{u}_{-1}, \mathrm{u}_{0}, \mathrm{u}_{1}, \mathrm{u}_{2}\right\}$ and $\left\{\mathrm{u}_{0}, \mathrm{u}_{1}, \mathrm{u}_{2}, \mathrm{u}_{3}\right\}$ respectively. The $i^{\text {th }}$ reconstructed polynomial corresponding to stencil $\mathrm{S}_{i}^{r 4}$ is then expressed as

$\mathrm{P}_{i}^{r 4}(x)=\mathrm{u}_{0}+\mathrm{u}_{x} \mathrm{~L}_{1}(x)+\mathrm{u}_{x 2} \mathrm{~L}_{2}(x)+\mathrm{u}_{x 3} \mathrm{~L}_{3}(x)$

The stencil $\mathrm{S}_{1}^{r 4}$ gives

$$
\begin{aligned}
& \mathrm{u}_{x}=\left(-177 \mathrm{u}_{-1}+87 \mathrm{u}_{-2}-19 \mathrm{u}_{-3}+109 \mathrm{u}_{0}\right) / 60 \\
& \mathrm{u}_{x 2}=-5 \mathrm{u}_{-1} / 2+2 \mathrm{u}_{-2}-\mathrm{u}_{-3} / 2+\mathrm{u}_{0} \\
& \mathrm{u}_{x 3}=\left(-3 \mathrm{u}_{-1}+3 \mathrm{u}_{-2}-\mathrm{u}_{-3}+\mathrm{u}_{0}\right) / 6
\end{aligned}
$$

The stencil $\mathrm{S}_{2}^{r 4}$ gives

$$
\begin{aligned}
& u_{x}=\left(-63 u_{-1}+11 u_{-2}+33 u_{0}+19 u_{1}\right) / 60 \\
& u_{x 2}=u_{-1} / 2-u_{0}+u_{1} / 2 \\
& u_{x 3}=\left(3 u_{-1}-u_{-2}-3 u_{0}+u_{1}\right) / 6
\end{aligned}
$$

The stencil $\mathrm{S}_{3}^{r 4}$ gives 
$\mathrm{u}_{x}=\left(-19 \mathrm{u}_{-1}-33 \mathrm{u}_{0}+63 \mathrm{u}_{1}-11 \mathrm{u}_{2}\right) / 60$,

$\mathrm{u}_{x 2}=\mathrm{u}_{-1} / 2-\mathrm{u}_{0}+\mathrm{u}_{1} / 2$,

$\mathrm{u}_{x 3}=\left(-\mathrm{u}_{-1}+3 \mathrm{u}_{0}-3 \mathrm{u}_{1}+\mathrm{u}_{2}\right) / 6$

The stencil $\mathrm{S}_{4}^{r 4}$ gives

$\mathrm{u}_{x}=\left(-109 \mathrm{u}_{0}+177 \mathrm{u}_{1}-87 \mathrm{u}_{2}+19 \mathrm{u}_{3}\right) / 60$,

$\mathrm{u}_{x 2}=\mathrm{u}_{0}-5 \mathrm{u}_{1} / 2+2 \mathrm{u}_{2}-\mathrm{u}_{3} / 2$,

$\mathrm{u}_{x 3}=\left(-\mathrm{u}_{0}+3 \mathrm{u}_{1}-3 \mathrm{u}_{2}+\mathrm{u}_{3}\right) / 6$

Because of the formulation in Legendre basis, the smoothness indicator for each of the four stencils has the same expression. Unlike the expressions given in Balsara \& Shu [1], it can be written as a sum of perfect squares as shown below

$\beta^{r 4}=\left(\mathrm{u}_{x}+\mathrm{u}_{x 3} / 10\right)^{2}+\frac{13}{3}\left(\mathrm{u}_{x 2}\right)^{2}+\frac{781}{20}\left(\mathrm{u}_{x 3}\right)^{2}$

For each stencil $\mathrm{S}_{i}^{r 4}$, we can add a subscript " $i$ " to the smoothness indicator in eqn. (2.12) to denote that it corresponds to a specific stencil. Notice that the above expression is considerably more compact that the corresponding expression in Balsara \& Shu [1], though the expressions are mathematically equivalent.

\section{II.3) $r=5$ WENO Reconstruction}

When considering the $r=5$ WENO reconstruction problem in zone " 0 ", we focus on the neighboring zone-averaged values $\left\{\mathrm{u}_{-4}, \mathrm{u}_{-3}, \mathrm{u}_{-2}, \mathrm{u}_{-1}, \mathrm{u}_{0}, \mathrm{u}_{1}, \mathrm{u}_{2}, \mathrm{u}_{3}, \mathrm{u}_{4}\right\}$. A fifth order reconstruction for the zone labeled " 0 " can be carried out by using five stencils $\mathrm{S}_{1}^{r 5}$, $\mathrm{S}_{2}^{r 5}, \mathrm{~S}_{3}^{r 5}, \mathrm{~S}_{4}^{r 5}$ and $\mathrm{S}_{5}^{r 5}$ that rely on the variables $\left\{\mathrm{u}_{-4}, \mathrm{u}_{-3}, \mathrm{u}_{-2}, \mathrm{u}_{-1}, \mathrm{u}_{0}\right\}$, $\left\{\mathrm{u}_{-3}, \mathrm{u}_{-2}, \mathrm{u}_{-1}, \mathrm{u}_{0}, \mathrm{u}_{1}\right\} \quad\left\{\mathrm{u}_{-2}, \mathrm{u}_{-1}, \mathrm{u}_{0}, \mathrm{u}_{1}, \mathrm{u}_{2}\right\}, \quad\left\{\mathrm{u}_{-1}, \mathrm{u}_{0}, \mathrm{u}_{1}, \mathrm{u}_{2}, \mathrm{u}_{3}\right\}$ and $\left\{\mathrm{u}_{0}, \mathrm{u}_{1}, \mathrm{u}_{2}, \mathrm{u}_{3}, \mathrm{u}_{4}\right\}$ 
respectively. The $i^{\text {th }}$ reconstructed polynomial corresponding to stencil $\mathrm{S}_{i}^{r 5}$ is then expressed as

$\mathrm{P}_{i}^{r 5}(x)=\mathrm{u}_{0}+\mathrm{u}_{x} \mathrm{~L}_{1}(x)+\mathrm{u}_{x 2} \mathrm{~L}_{2}(x)+\mathrm{u}_{x 3} \mathrm{~L}_{3}(x)+\mathrm{u}_{x 4} \mathrm{~L}_{4}(x)$

The stencil $\mathrm{S}_{1}^{r 5}$ gives

$\mathrm{u}_{x}=\left(-462 \mathrm{u}_{-1}+336 \mathrm{u}_{-2}-146 \mathrm{u}_{-3}+27 \mathrm{u}_{-4}+245 \mathrm{u}_{0}\right) / 120$,

$\mathrm{u}_{x 2}=\left(-240 \mathrm{u}_{-1}+262 \mathrm{u}_{-2}-128 \mathrm{u}_{-3}+25 \mathrm{u}_{-4}+81 \mathrm{u}_{0}\right) / 56$,

$\mathrm{u}_{x 3}=\left(-18 \mathrm{u}_{-1}+24 \mathrm{u}_{-2}-14 \mathrm{u}_{-3}+3 \mathrm{u}_{-4}+5 \mathrm{u}_{0}\right) / 12$,

$\mathrm{u}_{x 4}=\left(-4 \mathrm{u}_{-1}+6 \mathrm{u}_{-2}-4 \mathrm{u}_{-3}+\mathrm{u}_{-4}+\mathrm{u}_{0}\right) / 24$

The stencil $\mathrm{S}_{2}^{r 5}$ gives

$\mathrm{u}_{x}=\left(-192 \mathrm{u}_{-1}+66 \mathrm{u}_{-2}-11 \mathrm{u}_{-3}+110 \mathrm{u}_{0}+27 \mathrm{u}_{1}\right) / 120$,

$\mathrm{u}_{x 2}=\left(10 \mathrm{u}_{-1}+12 \mathrm{u}_{-2}-3 \mathrm{u}_{-3}-44 \mathrm{u}_{0}+25 \mathrm{u}_{1}\right) / 56$,

$u_{x 3}=\left(12 u_{-1}-6 u_{-2}+u_{-3}-10 u_{0}+3 u_{1}\right) / 12$,

$\mathrm{u}_{x 4}=\left(6 \mathrm{u}_{-1}-4 \mathrm{u}_{-2}+\mathrm{u}_{-3}-4 \mathrm{u}_{0}+\mathrm{u}_{1}\right) / 24$

The stencil $\mathrm{S}_{3}^{r 5}$ gives

$\mathrm{u}_{x}=\left(-82 \mathrm{u}_{-1}+11 \mathrm{u}_{-2}+82 \mathrm{u}_{1}-11 \mathrm{u}_{2}\right) / 120$,

$u_{x 2}=\left(40 u_{-1}-3 u_{-2}-74 u_{0}+40 u_{1}-3 u_{2}\right) / 56$,

$\mathrm{u}_{x 3}=\left(2 \mathrm{u}_{-1}-\mathrm{u}_{-2}-2 \mathrm{u}_{1}+\mathrm{u}_{2}\right) / 12$,

$\mathrm{u}_{x 4}=\left(-4 \mathrm{u}_{-1}+\mathrm{u}_{-2}+6 \mathrm{u}_{0}-4 \mathrm{u}_{1}+\mathrm{u}_{2}\right) / 24$

The stencil $\mathrm{S}_{4}^{r 5}$ gives 
$\mathrm{u}_{x}=\left(-27 \mathrm{u}_{-1}-110 \mathrm{u}_{0}+192 \mathrm{u}_{1}-66 \mathrm{u}_{2}+11 \mathrm{u}_{3}\right) / 120$,

$u_{x 2}=\left(25 u_{-1}-44 u_{0}+10 u_{1}+12 u_{2}-3 u_{3}\right) / 56$,

$\mathrm{u}_{x 3}=\left(-3 \mathrm{u}_{-1}+10 \mathrm{u}_{0}-12 \mathrm{u}_{1}+6 \mathrm{u}_{2}-\mathrm{u}_{3}\right) / 12$,

$\mathrm{u}_{x 4}=\left(\mathrm{u}_{-1}-4 \mathrm{u}_{0}+6 \mathrm{u}_{1}-4 \mathrm{u}_{2}+\mathrm{u}_{3}\right) / 24$

The stencil $S_{5}^{r 5}$ gives

$\mathrm{u}_{x}=\left(-245 \mathrm{u}_{0}+462 \mathrm{u}_{1}-336 \mathrm{u}_{2}+146 \mathrm{u}_{3}-27 \mathrm{u}_{4}\right) / 120$,

$u_{x 2}=\left(81 u_{0}-240 u_{1}+262 u_{2}-128 u_{3}+25 u_{4}\right) / 56$,

$\mathrm{u}_{x 3}=\left(-5 \mathrm{u}_{0}+18 \mathrm{u}_{1}-24 \mathrm{u}_{2}+14 \mathrm{u}_{3}-3 \mathrm{u}_{4}\right) / 12$,

$\mathrm{u}_{x 4}=\left(\mathrm{u}_{0}-4 \mathrm{u}_{1}+6 \mathrm{u}_{2}-4 \mathrm{u}_{3}+\mathrm{u}_{4}\right) / 24$

The smoothness indicator for each of the five stencils has the same expression and can be written as

$\beta^{r 5}=\left(\mathrm{u}_{x}+\mathrm{u}_{x 3} / 10\right)^{2}+\frac{13}{3}\left(\mathrm{u}_{x 2}+\frac{123}{455} \mathrm{u}_{x 4}\right)^{2}+\frac{781}{20}\left(\mathrm{u}_{x 3}\right)^{2}+\frac{1421461}{2275}\left(\mathrm{u}_{x 4}\right)^{2}$

For each stencil $S_{i}^{r 5}$, we can add a subscript " $i$ " to the smoothness indicator in eqn. (2.19) to denote that it corresponds to a specific stencil. Notice that eqn. (2.19) is also a sum of perfect squares. Furthermore, compare eqn. (2.19) to eqn. (2.12) to observe that all the moments up to $u_{x 3}$ carry the same coefficients in the two equations. The two equations only differ by terms involving the moment $u_{x 4}$ in such a way that all the lower moments, i.e. $u_{x}, u_{x 2}$ and $u_{x 3}$, have the same coefficients. This illustrates the general pattern that emerges when constructing smoothness indicators in Legendre basis. The smoothness indicator for each new value of " $r$ " differs from the smoothness indicator for " $r-1$ " by terms that only involve the newest moment that is added. We will see this pattern borne out in the next Sub-section. 
It is also worth pointing out that the central stencil $\mathrm{S}_{3}^{r 5}$ contains all the zones that would be used for the composite stencil of the $r=3$ WENO. This property will be utilized later to design WENO schemes with adaptive order. In other words, a suitable non-linear hybridization between the stencil $\mathrm{S}_{3}^{r 5}$ and the three CWENO stencils with $r=3$ will give us a WENO-AO $(5,3)$ reconstruction strategy with an adaptive order that can range between $5^{\text {th }}$ order (at best) and $3^{\text {rd }}$ order (at worst). When a $5^{\text {th }}$ order reconstruction using the stencil $\mathrm{S}_{3}^{r 5}$ is likely to become excessively oscillatory, the non-linear hybridization will enable the scheme to switch away to a third order CWENO scheme which is known to have excellent stability properties as well as an ability to preserve extrema.

\section{II.4) $r=6$ WENO Reconstruction}

When considering the $r=6$ WENO reconstruction problem in zone " 0 ", we focus on the neighboring zone-averaged values $\left\{\mathrm{u}_{-5}, \mathrm{u}_{-4}, \mathrm{u}_{-3}, \mathrm{u}_{-2}, \mathrm{u}_{-1}, \mathrm{u}_{0}, \mathrm{u}_{1}, \mathrm{u}_{2}, \mathrm{u}_{3}, \mathrm{u}_{4}, \mathrm{u}_{5}\right\}$. A sixth order reconstruction for the zone labeled " 0 " can be carried out by using five stencils $\mathrm{S}_{1}^{r 6}, \mathrm{~S}_{2}^{r 6}, \mathrm{~S}_{3}^{r 6}, \mathrm{~S}_{4}^{r 6}, \mathrm{~S}_{5}^{r 6}$ and $\mathrm{S}_{6}^{r 6}$ that rely on the variables $\left\{\mathrm{u}_{-5}, \mathrm{u}_{-4}, \mathrm{u}_{-3}, \mathrm{u}_{-2}, \mathrm{u}_{-1}, \mathrm{u}_{0}\right\} \quad, \quad\left\{\mathrm{u}_{-4}, \mathrm{u}_{-3}, \mathrm{u}_{-2}, \mathrm{u}_{-1}, \mathrm{u}_{0}, \mathrm{u}_{1}\right\} \quad\left\{\mathrm{u}_{-3}, \mathrm{u}_{-2}, \mathrm{u}_{-1}, \mathrm{u}_{0}, \mathrm{u}_{1}, \mathrm{u}_{2}\right\} \quad$, $\left\{\mathrm{u}_{-2}, \mathrm{u}_{-1}, \mathrm{u}_{0}, \mathrm{u}_{1}, \mathrm{u}_{2}, \mathrm{u}_{3}\right\},\left\{\mathrm{u}_{-1}, \mathrm{u}_{0}, \mathrm{u}_{1}, \mathrm{u}_{2}, \mathrm{u}_{3}, \mathrm{u}_{4}\right\}$ and $\left\{\mathrm{u}_{0}, \mathrm{u}_{1}, \mathrm{u}_{2}, \mathrm{u}_{3}, \mathrm{u}_{4}, \mathrm{u}_{5}\right\}$ respectively. The $i^{\text {th }}$ reconstructed polynomial corresponding to stencil $\mathrm{S}_{i}^{r 6}$ is then expressed as

$$
\mathrm{P}_{i}^{r 6}(x)=\mathrm{u}_{0}+\mathrm{u}_{x} \mathrm{~L}_{1}(x)+\mathrm{u}_{x 2} \mathrm{~L}_{2}(x)+\mathrm{u}_{x 3} \mathrm{~L}_{3}(x)+\mathrm{u}_{x 4} \mathrm{~L}_{4}(x)+\mathrm{u}_{x 5} \mathrm{~L}_{5}(x)
$$

The stencil $\mathrm{S}_{1}^{r 6}$ gives 


$$
\begin{aligned}
& \mathrm{u}_{x}=\left(-23719 \mathrm{u}_{-1}+22742 \mathrm{u}_{-2}-14762 \mathrm{u}_{-3}+5449 \mathrm{u}_{-4}-863 \mathrm{u}_{-5}+11153 \mathrm{u}_{0}\right) / 5040 \\
& \mathrm{u}_{x 2}=\left(-350 \mathrm{u}_{-1}+482 \mathrm{u}_{-2}-348 \mathrm{u}_{-3}+135 \mathrm{u}_{-4}-22 \mathrm{u}_{-5}+103 \mathrm{u}_{0}\right) / 56 \\
& \mathrm{u}_{x 3}=\left(-317 \mathrm{u}_{-1}+526 \mathrm{u}_{-2}-436 \mathrm{u}_{-3}+182 \mathrm{u}_{-4}-31 \mathrm{u}_{-5}+76 \mathrm{u}_{0}\right) / 108 \\
& \mathrm{u}_{x 4}=\left(-14 \mathrm{u}_{-1}+26 \mathrm{u}_{-2}-24 \mathrm{u}_{-3}+11 \mathrm{u}_{-4}-2 \mathrm{u}_{-5}+3 \mathrm{u}_{0}\right) / 24 \\
& \mathrm{u}_{x 5}=\left(-5 \mathrm{u}_{-1}+10 \mathrm{u}_{-2}-10 \mathrm{u}_{-3}+5 \mathrm{u}_{-4}-\mathrm{u}_{-5}+\mathrm{u}_{0}\right) / 120
\end{aligned}
$$

The stencil $\mathrm{S}_{2}^{r 6}$ gives

$$
\begin{aligned}
& \mathrm{u}_{x}=\left(-10774 \mathrm{u}_{-1}+5482 \mathrm{u}_{-2}-1817 \mathrm{u}_{-3}+271 \mathrm{u}_{-4}+5975 \mathrm{u}_{0}+863 \mathrm{u}_{1}\right) / 5040 \\
& \mathrm{u}_{x 2}=\left(-20 \mathrm{u}_{-1}+42 \mathrm{u}_{-2}-18 \mathrm{u}_{-3}+3 \mathrm{u}_{-4}-29 \mathrm{u}_{0}+22 \mathrm{u}_{1}\right) / 56 \\
& \mathrm{u}_{x 3}=\left(148 \mathrm{u}_{-1}-94 \mathrm{u}_{-2}+29 \mathrm{u}_{-3}-4 \mathrm{u}_{-4}-110 \mathrm{u}_{0}+31 \mathrm{u}_{1}\right) / 108 \\
& \mathrm{u}_{x 4}=\left(16 \mathrm{u}_{-1}-14 \mathrm{u}_{-2}+6 \mathrm{u}_{-3}-\mathrm{u}_{-4}-9 \mathrm{u}_{0}+2 \mathrm{u}_{1}\right) / 24 \\
& \mathrm{u}_{x 5}=\left(10 \mathrm{u}_{-1}-10 \mathrm{u}_{-2}+5 \mathrm{u}_{-3}-\mathrm{u}_{-4}-5 \mathrm{u}_{0}+\mathrm{u}_{1}\right) / 120
\end{aligned}
$$

The stencil $\mathrm{S}_{3}^{r 6}$ gives

$$
\begin{aligned}
& \mathrm{u}_{x}=\left(-5354 \mathrm{u}_{-1}+1417 \mathrm{u}_{-2}-191 \mathrm{u}_{-3}+1910 \mathrm{u}_{0}+2489 \mathrm{u}_{1}-271 \mathrm{u}_{2}\right) / 5040, \\
& \mathrm{u}_{x 2}=\left(40 \mathrm{u}_{-1}-3 \mathrm{u}_{-2}-74 \mathrm{u}_{0}+40 \mathrm{u}_{1}-3 \mathrm{u}_{2}\right) / 56 \\
& \mathrm{u}_{x 3}=\left(68 \mathrm{u}_{-1}-34 \mathrm{u}_{-2}+5 \mathrm{u}_{-3}-50 \mathrm{u}_{0}+7 \mathrm{u}_{1}+4 \mathrm{u}_{2}\right) / 108 \\
& \mathrm{u}_{x 4}=\left(-4 \mathrm{u}_{-1}+\mathrm{u}_{-2}+6 \mathrm{u}_{0}-4 \mathrm{u}_{1}+\mathrm{u}_{2}\right) / 24 \\
& \mathrm{u}_{x 5}=\left(-10 \mathrm{u}_{-1}+5 \mathrm{u}_{-2}-\mathrm{u}_{-3}+10 \mathrm{u}_{0}-5 \mathrm{u}_{1}+\mathrm{u}_{2}\right) / 120
\end{aligned}
$$

The stencil $\mathrm{S}_{4}^{r 6}$ gives

$$
\begin{aligned}
& \mathrm{u}_{x}=\left(-2489 \mathrm{u}_{-1}+271 \mathrm{u}_{-2}-1910 \mathrm{u}_{0}+5354 \mathrm{u}_{1}-1417 \mathrm{u}_{2}+191 \mathrm{u}_{3}\right) / 5040 \\
& \mathrm{u}_{x 2}=\left(40 \mathrm{u}_{-1}-3 \mathrm{u}_{-2}-74 \mathrm{u}_{0}+40 \mathrm{u}_{1}-3 \mathrm{u}_{2}\right) / 56 \\
& \mathrm{u}_{x 3}=\left(-7 \mathrm{u}_{-1}-4 \mathrm{u}_{-2}+50 \mathrm{u}_{0}-68 \mathrm{u}_{1}+34 \mathrm{u}_{2}-5 \mathrm{u}_{3}\right) / 108 \\
& \mathrm{u}_{x 4}=\left(-4 \mathrm{u}_{-1}+\mathrm{u}_{-2}+6 \mathrm{u}_{0}-4 \mathrm{u}_{1}+\mathrm{u}_{2}\right) / 24 \\
& \mathrm{u}_{x 5}=\left(5 \mathrm{u}_{-1}-\mathrm{u}_{-2}-10 \mathrm{u}_{0}+10 \mathrm{u}_{1}-5 \mathrm{u}_{2}+\mathrm{u}_{3}\right) / 120
\end{aligned}
$$


The stencil $S_{5}^{r 6}$ gives

$$
\begin{aligned}
& \mathrm{u}_{x}=\left(-863 \mathrm{u}_{-1}-5975 \mathrm{u}_{0}+10774 \mathrm{u}_{1}-5482 \mathrm{u}_{2}+1817 \mathrm{u}_{3}-271 \mathrm{u}_{4}\right) / 5040 \\
& \mathrm{u}_{x 2}=\left(22 \mathrm{u}_{-1}-29 \mathrm{u}_{0}-20 \mathrm{u}_{1}+42 \mathrm{u}_{2}-18 \mathrm{u}_{3}+3 \mathrm{u}_{4}\right) / 56 \\
& \mathrm{u}_{x 3}=\left(-31 \mathrm{u}_{-1}+110 \mathrm{u}_{0}-148 \mathrm{u}_{1}+94 \mathrm{u}_{2}-29 \mathrm{u}_{3}+4 \mathrm{u}_{4}\right) / 108 \\
& \mathrm{u}_{x 4}=\left(2 \mathrm{u}_{-1}-9 \mathrm{u}_{0}+16 \mathrm{u}_{1}-14 \mathrm{u}_{2}+6 \mathrm{u}_{3}-\mathrm{u}_{4}\right) / 24 \\
& \mathrm{u}_{x 5}=\left(-\mathrm{u}_{-1}+5 \mathrm{u}_{0}-10 \mathrm{u}_{1}+10 \mathrm{u}_{2}-5 \mathrm{u}_{3}+\mathrm{u}_{4}\right) / 120
\end{aligned}
$$

The stencil $\mathrm{S}_{6}^{r 6}$ gives

$$
\begin{aligned}
& \mathrm{u}_{x}=\left(-11153 \mathrm{u}_{0}+23719 \mathrm{u}_{1}-22742 \mathrm{u}_{2}+14762 \mathrm{u}_{3}-5449 \mathrm{u}_{4}+863 \mathrm{u}_{5}\right) / 5040, \\
& \mathrm{u}_{x 2}=\left(103 \mathrm{u}_{0}-350 \mathrm{u}_{1}+482 \mathrm{u}_{2}-348 \mathrm{u}_{3}+135 \mathrm{u}_{4}-22 \mathrm{u}_{5}\right) / 56, \\
& \mathrm{u}_{x 3}=\left(-76 \mathrm{u}_{0}+317 \mathrm{u}_{1}-526 \mathrm{u}_{2}+436 \mathrm{u}_{3}-182 \mathrm{u}_{4}+31 \mathrm{u}_{5}\right) / 108, \\
& \mathrm{u}_{x 4}=\left(3 \mathrm{u}_{0}-14 \mathrm{u}_{1}+26 \mathrm{u}_{2}-24 \mathrm{u}_{3}+11 \mathrm{u}_{4}-2 \mathrm{u}_{5}\right) / 24, \\
& \mathrm{u}_{x 5}=\left(-\mathrm{u}_{0}+5 \mathrm{u}_{1}-10 \mathrm{u}_{2}+10 \mathrm{u}_{3}-5 \mathrm{u}_{4}+\mathrm{u}_{5}\right) / 120
\end{aligned}
$$

The smoothness indicator for each of the six stencils has the same expression and can be written as

$$
\begin{aligned}
\beta^{r 6} & =\left(\mathrm{u}_{x}+\mathrm{u}_{x 3} / 10+\mathrm{u}_{x 5} / 126\right)^{2}+\frac{13}{3}\left(\mathrm{u}_{x 2}+\frac{123}{455} \mathrm{u}_{x 4}\right)^{2}+\frac{781}{20}\left(\mathrm{u}_{x 3}+\frac{26045}{49203} \mathrm{u}_{x 5}\right)^{2} \\
+ & \frac{1421461}{2275}\left(\mathrm{u}_{x 4}\right)^{2}+\frac{21520059541}{1377684}\left(\mathrm{u}_{x 5}\right)^{2}
\end{aligned}
$$

For each stencil $S_{i}^{r 6}$, we can add a subscript " $i$ " to the smoothness indicator in eqn. (2.27) to denote that it corresponds to a specific stencil. As before, the smoothness indicator can be written as a sum of perfect squares. Furthermore, on comparing eqns. (2.19) and (2.27) 
we see that the additional terms in eqn. (2.27) simply involve $\mathrm{u}_{x 5}$, with the lower moments retaining the same coefficients.

\section{II.5) $r=7$ WENO Reconstruction}

When considering the $r=7$ WENO reconstruction problem in zone " 0 ", we focus on the neighboring zone-averaged values $\left\{\mathrm{u}_{-6}, \mathrm{u}_{-5}, \mathrm{u}_{-4}, \mathrm{u}_{-3}, \mathrm{u}_{-2}, \mathrm{u}_{-1}, \mathrm{u}_{0}, \mathrm{u}_{1}, \mathrm{u}_{2}, \mathrm{u}_{3}, \mathrm{u}_{4}, \mathrm{u}_{5}, \mathrm{u}_{6}\right\}$. A seventh order reconstruction for the zone labeled " 0 " can be carried out by using seven stencils $S_{1}^{r 7}$ to $S_{7}^{r 7}$. Since the logic for constructing these stencils is now transparent, we will not explicitly catalogue them. Only the central stencil $\mathrm{S}_{4}^{r 7}$, which relies on the variables $\left\{\mathrm{u}_{-3}, \mathrm{u}_{-2}, \mathrm{u}_{-1}, \mathrm{u}_{0}, \mathrm{u}_{1}, \mathrm{u}_{2}, \mathrm{u}_{3}\right\}$, is useful in our further discussions. The $i^{\text {th }}$ reconstructed polynomial corresponding to stencil $\mathrm{S}_{i}^{r 7}$ is then expressed as

$$
\begin{aligned}
\mathrm{P}_{i}^{r 7}(x) & =\mathrm{u}_{0}+\mathrm{u}_{x} \mathrm{~L}_{1}(x)+\mathrm{u}_{x 2} \mathrm{~L}_{2}(x)+\mathrm{u}_{x 3} \mathrm{~L}_{3}(x)+\mathrm{u}_{x 4} \mathrm{~L}_{4}(x)+\mathrm{u}_{x 5} \mathrm{~L}_{5}(x) \\
& +\mathrm{u}_{x 6} \mathrm{~L}_{6}(x)
\end{aligned}
$$

The central stencil $\mathrm{S}_{4}^{r 7}$ gives

$$
\begin{aligned}
& \mathrm{u}_{x}=\left(-7843 \mathrm{u}_{-1}+1688 \mathrm{u}_{-2}-191 \mathrm{u}_{-3}+7843 \mathrm{u}_{1}-1688 \mathrm{u}_{2}+191 \mathrm{u}_{3}\right) / 10080 \\
& \mathrm{u}_{x 2}=\left(8385 \mathrm{u}_{-1}-1014 \mathrm{u}_{-2}+79 \mathrm{u}_{-3}-14900 \mathrm{u}_{0}+8385 \mathrm{u}_{1}-1014 \mathrm{u}_{2}+79 \mathrm{u}_{3}\right) / 10080 \\
& \mathrm{u}_{x 3}=\left(61 \mathrm{u}_{-1}-38 \mathrm{u}_{-2}+5 \mathrm{u}_{-3}-61 \mathrm{u}_{1}+38 \mathrm{u}_{2}-5 \mathrm{u}_{3}\right) / 216 \\
& \mathrm{u}_{x 4}=\left(-459 \mathrm{u}_{-1}+144 \mathrm{u}_{-2}-13 \mathrm{u}_{-3}+656 \mathrm{u}_{0}-459 \mathrm{u}_{1}+144 \mathrm{u}_{2}-13 \mathrm{u}_{3}\right) / 1584 \\
& \mathrm{u}_{x 5}=\left(-5 \mathrm{u}_{-1}+4 \mathrm{u}_{-2}-\mathrm{u}_{-3}+5 \mathrm{u}_{1}-4 \mathrm{u}_{2}+\mathrm{u}_{3}\right) / 240 \\
& \mathrm{u}_{x 6}=\left(15 \mathrm{u}_{-1}-6 \mathrm{u}_{-2}+\mathrm{u}_{-3}-20 \mathrm{u}_{0}+15 \mathrm{u}_{1}-6 \mathrm{u}_{2}+\mathrm{u}_{3}\right) / 720
\end{aligned}
$$

The analogous expressions for the other six stencils for the $r=7$ polynomials can be obtained by following a reconstruction via primitive function approach on a computer algebra system. We restrict ourselves to explicitly cataloguing $\mathrm{S}_{4}^{r 7}$ because it is the only 
stencil that contributes to WENO-AO(7,3). The smoothness indicator for each of the seven stencils has the same expression and can be written as

$$
\begin{aligned}
\beta^{r 7} & =\left(u_{x}+u_{x 3} / 10+u_{x 5} / 126\right)^{2}+\frac{13}{3}\left(u_{x 2}+\frac{123}{455} u_{x 4}+\frac{85}{2002} u_{x 6}\right)^{2} \\
& +\frac{781}{20}\left(u_{x 3}+\frac{26045}{49203} u_{x 5}\right)^{2}+\frac{1421461}{2275}\left(u_{x 4}+\frac{81596225}{93816426} u_{x 6}\right)^{2} \\
& +\frac{21520059541}{1377684}\left(u_{x 5}\right)^{2}+\frac{15510384942580921}{27582029244}\left(u_{x 6}\right)^{2}
\end{aligned}
$$

For each stencil $S_{i}^{r 7}$, we can add a subscript " $i$ " to the smoothness indicator in eqn. (2.30) to denote that it corresponds to a specific stencil. As before, the smoothness indicator can be written as a sum of perfect squares. Furthermore, on comparing eqns. (2.27) and (2.30) we see that the additional terms in eqn. (2.30) simply involve $\mathrm{u}_{x 6}$, with the lower moments retaining the same coefficients.

It is also worth pointing out that the central stencil $\mathrm{S}_{4}^{r 7}$ contains all the zones that would be used for the composite stencil of the $r=4$ WENO. This property will be utilized later to design WENO schemes with adaptive order. In other words, a suitable non-linear hybridization between the stencil $\mathrm{S}_{4}^{r 7}$ and the three CWENO stencils with $r=3$ will give us a WENO-AO $(7,3)$ reconstruction strategy with an adaptive order that can range between $7^{\text {th }}$ order (at best) and $3^{\text {rd }}$ order (at worst). When a $7^{\text {th }}$ order reconstruction using the stencil $\mathrm{S}_{4}^{r 7}$ is likely to become excessively oscillatory, the non-linear hybridization will enable the scheme to switch away to a third order CWENO which is known to have excellent stability properties as well as an ability to preserve extrema.

Having read the previous paragraph it is also possible to realize that one can recursively make a suitable non-linear hybridization between the non-linearly stabilized WENO-AO(7,3) reconstruction strategy and its smaller cousin, the non-linearly stabilized WENO-AO(5,3) reconstruction strategy. This gives us a $100 \%$ stable strategy of 
switching from a seventh order scheme to a fifth order scheme and further switching from fifth order to third order.

\section{II.6) $r=8$ WENO Reconstruction}

When considering the $r=8$ WENO reconstruction problem in zone " 0 ", we focus on the neighboring zone-averaged values $\left\{\mathrm{u}_{-7}, \mathrm{u}_{-6}, \mathrm{u}_{-5}, \mathrm{u}_{-4}, \mathrm{u}_{-3}, \mathrm{u}_{-2}, \mathrm{u}_{-1}, \mathrm{u}_{0}, \mathrm{u}_{1}, \mathrm{u}_{2}, \mathrm{u}_{3}, \mathrm{u}_{4}, \mathrm{u}_{5}, \mathrm{u}_{6}, \mathrm{u}_{7}\right\}$. An eighth order reconstruction for the zone labeled " 0 " can be carried out by using eight stencils. Since the logic for constructing these stencils is now transparent, we will not explicitly catalogue them. The further reason for not explicitly cataloguing them is, of course, that the stencils for $r=8$ WENO do not have a central stencil. As a result, we cannot use the central stencil to construct a WENO-AO $(8,3)$ scheme. For that reason, we content ourselves with documenting the $i^{\text {th }}$ reconstructed polynomial corresponding to stencil $\mathrm{S}_{i}^{r 8}$ which is expressed as

$$
\begin{aligned}
\mathrm{P}_{i}^{r 8}(x) & =\mathrm{u}_{0}+\mathrm{u}_{x} \mathrm{~L}_{1}(x)+\mathrm{u}_{x 2} \mathrm{~L}_{2}(x)+\mathrm{u}_{x 3} \mathrm{~L}_{3}(x)+\mathrm{u}_{x 4} \mathrm{~L}_{4}(x)+\mathrm{u}_{x 5} \mathrm{~L}_{5}(x) \\
& +\mathrm{u}_{x 6} \mathrm{~L}_{6}(x)+\mathrm{u}_{x 7} \mathrm{~L}_{7}(x)
\end{aligned}
$$

The corresponding smoothness indicator for each of the eight stencils has the same expression and can be written as 


$$
\begin{aligned}
\beta^{r 8} & =\left(u_{x}+u_{x 3} / 10+u_{x 5} / 126+u_{x 7} / 1716\right)^{2} \\
& +\frac{13}{3}\left(u_{x 2}+\frac{123}{455} u_{x 4}+\frac{85}{2002} u_{x 6}\right)^{2} \\
+ & \frac{781}{20}\left(u_{x 3}+\frac{26045}{49203} u_{x 5}+\frac{8395}{60918} u_{x 7}\right)^{2} \\
+ & \frac{1421461}{2275}\left(u_{x 4}+\frac{81596225}{93816426} u_{x 6}\right)^{2} \\
+ & \frac{21520059541}{1377684}\left(u_{x 5}+\frac{722379670131}{559521548066} u_{x 7}\right)^{2} \\
+ & \frac{15510384942580921}{27582029244}\left(u_{x 6}\right)^{2}+\frac{12210527897166191835083}{443141066068272}\left(u_{x 7}\right)^{2}
\end{aligned}
$$

For each stencil $S_{i}^{r 8}$, we can add a subscript " $i$ " to the smoothness indicator in eqn. (2.32) to denote that it corresponds to a specific stencil. As before, the smoothness measure can be written as a sum of perfect squares. Furthermore, on comparing eqns. (2.30) and (2.32) we see that the additional terms in eqn. (2.32) simply involve $u_{x 7}$, with the lower moments retaining the same coefficients.

\section{II.7) $r=9$ WENO Reconstruction}

When considering the $r=9$ WENO reconstruction problem in zone " 0 ", we focus on the neighboring zone-averaged values $\left\{\mathrm{u}_{-8}, \mathrm{u}_{-7}, \mathrm{u}_{-6}, \mathrm{u}_{-5}, \mathrm{u}_{-4}, \mathrm{u}_{-3}, \mathrm{u}_{-2}, \mathrm{u}_{-1}, \mathrm{u}_{0}, \mathrm{u}_{1}, \mathrm{u}_{2}, \mathrm{u}_{3}, \mathrm{u}_{4}, \mathrm{u}_{5}, \mathrm{u}_{6}, \mathrm{u}_{7}, \mathrm{u}_{8}\right\}$. A ninth order reconstruction for the zone labeled " 0 " can be carried out by using nine stencils $\mathrm{S}_{1}^{r 9}$ to $\mathrm{S}_{9}^{r 9}$. Since the logic for constructing these stencils is now transparent, we will not explicitly catalogue them. Only the central stencil $\mathrm{S}_{5}^{r 9}$, which relies on the variables $\left\{\mathrm{u}_{-4}, \mathrm{u}_{-3}, \mathrm{u}_{-2}, \mathrm{u}_{-1}, \mathrm{u}_{0}, \mathrm{u}_{1}, \mathrm{u}_{2}, \mathrm{u}_{3}, \mathrm{u}_{4}\right\}$, is useful to our further discussions. The $i^{\text {th }}$ reconstructed polynomial corresponding to stencil $\mathrm{S}_{i}^{r 9}$ is then expressed as 


$$
\begin{aligned}
\mathrm{P}_{i}^{r 9}(x) & =\mathrm{u}_{0}+\mathrm{u}_{x} \mathrm{~L}_{1}(x)+\mathrm{u}_{x 2} \mathrm{~L}_{2}(x)+\mathrm{u}_{x 3} \mathrm{~L}_{3}(x)+\mathrm{u}_{x 4} \mathrm{~L}_{4}(x)+\mathrm{u}_{x 5} \mathrm{~L}_{5}(x) \\
& +\mathrm{u}_{x 6} \mathrm{~L}_{6}(x)+\mathrm{u}_{x 7} \mathrm{~L}_{7}(x)+\mathrm{u}_{x 8} \mathrm{~L}_{8}(x)
\end{aligned}
$$

The central stencil $\mathrm{S}_{5}^{r 9}$ gives

$$
\begin{aligned}
& u_{x}=\left(\begin{array}{l}
-505538 u_{-1}+136238 u_{-2}-26442 u_{-3}+2497 u_{-4}+505538 u_{1} \\
-136238 u_{2}+26442 u_{3}-2497 u_{4}
\end{array}\right) / 604800, \\
& u_{x 2}=\left(\begin{array}{l}
1205324 u_{-1}-183100 u_{-2}+24500 u_{-3}-1759 u_{-4}-2089930 u_{0} \\
+1205324 u_{1}-183100 u_{2}+24500 u_{3}-1759 u_{4}
\end{array}\right) / 1330560, \\
& u_{x 3}=\left(\begin{array}{l}
34414 u_{-1}-24294 u_{-2}+5446 u_{-3}-541 u_{-4}-34414 u_{1} \\
+24294 u_{2}-5446 u_{3}+541 u_{4}
\end{array}\right) / 95040, \\
& u_{x 4}=\left(\begin{array}{l}
-186496 u_{-1}+66572 u_{-2}-10240 u_{-3}+773 u_{-4}+258782 u_{0} \\
-186496 u_{1}+66572 u_{2}-10240 u_{3}+773 u_{4}
\end{array}\right) / 494208, \\
& u_{x 5}=\left(\begin{array}{l}
-526 u_{-1}+474 u_{-2}-166 u_{-3}+19 u_{-4}+526 u_{1} \\
-474 u_{2}+166 u_{3}-19 u_{4}
\end{array}\right) / 12480 \\
& u_{x 6}=\left(\begin{array}{l}
1852 u_{-1}-836 u_{-2}+196 u_{-3}-17 u_{-4}-2390 u_{0} \\
+1852 u_{1}-836 u_{2}+196 u_{3}-17 u_{4}
\end{array}\right) / 43200 \\
& u_{x 7}=\left(\begin{array}{l}
14 u_{-1}-14 u_{-2}+6 u_{-3}-u_{-4}-14 u_{1} \\
+14 u_{2}-6 u_{3}+u_{4}
\end{array}\right) / 10080 \\
& u_{x 8}=\left(\begin{array}{l}
-56 u_{-1}+28 u_{-2}-8 u_{-3}+u_{-4}+70 u_{0} \\
-56 u_{1}+28 u_{2}-8 u_{3}+u_{4}
\end{array}\right) / 40320
\end{aligned}
$$

The smoothness indicator for each of the nine stencils has the same expression and can be written as 


$$
\begin{aligned}
\beta^{r 9} & =\left(\mathrm{u}_{x}+\mathrm{u}_{x 3} / 10+\mathrm{u}_{x 5} / 126+\mathrm{u}_{x 7} / 1716\right)^{2} \\
+ & \frac{13}{3}\left(\mathrm{u}_{x 2}+\frac{123}{455} \mathrm{u}_{x 4}+\frac{85}{2002} \mathrm{u}_{x 6}+\frac{29}{5577} \mathrm{u}_{x 8}\right)^{2} \\
+ & \frac{781}{20}\left(\mathrm{u}_{x 3}+\frac{26045}{49203} \mathrm{u}_{x 5}+\frac{8395}{60918} \mathrm{u}_{x 7}\right)^{2} \\
+ & \frac{1421461}{2275}\left(\mathrm{u}_{x 4}+\frac{81596225}{93816426} \mathrm{u}_{x 6}+\frac{618438835}{1829420307} \mathrm{u}_{x 8}\right)^{2} \\
+ & \frac{21520059541}{1377684}\left(\mathrm{u}_{x 5}+\frac{722379670131}{559521548066} \mathrm{u}_{x 7}\right)^{2} \\
+ & \frac{15510384942580921}{27582029244}\left(\mathrm{u}_{x 6}+\frac{5423630339859998294}{3024525063803279595} \mathrm{u}_{x 8}\right)^{2} \\
+ & \frac{12210527897166191835083}{443141066068272}\left(\mathrm{u}_{x 7}\right)^{2}+\frac{75509368098103789336083731407561}{42818201328263029226415}\left(\mathrm{u}_{x 8}\right)^{2}
\end{aligned}
$$

For each stencil $\mathrm{S}_{i}^{r 9}$, we can add a subscript " $i$ " to the smoothness indicator in eqn. (2.35) to denote that it corresponds to a specific stencil. As before, the smoothness indicator can be written as a sum of perfect squares. Furthermore, on comparing eqns. (2.32) and (2.35) we see that the additional terms in eqn. (2.35) simply involve $\mathrm{u}_{x 8}$, with the lower moments retaining the same coefficients.

\section{III) WENO Schemes with Adaptive Order (WENO-AO)}

In this section we first describe some stage-setting associated with finite difference WENO schemes. Section III.1 then describes the fifth order accurate WENO$\mathrm{AO}(5,3)$ reconstruction. Section III.2 then describes the seventh order accurate WENO$\mathrm{AO}(7,3)$ reconstruction. Section III.3 then describes the formally ninth order accurate WENO-AO(9,3) reconstruction. Section III.4 then describes a hierarchical hybridization between the fifth order accurate WENO-AO $(5,3)$ reconstruction and the seventh order WENO-AO(7,3) reconstruction; we call it WENO-AO(7,5,3). The point of Section III.4 
is to show that the reconstruction improvement described here can be applied hierarchically and recursively.

It is easiest to briefly motivate finite difference WENO schemes in the following way. Consider the $\mathrm{N}$-component hyperbolic conservation law

$$
\frac{\partial \mathbf{U}}{\partial t}+\frac{\partial \mathbf{F}}{\partial x}=0
$$

For simplicity of presentation we focus on the one-dimensional case. For finite difference WENO formulations, treating multiple dimensions is easy because the same prescription for obtaining the numerical fluxes is applied to each of the dimensions and the gradients of the fluxes from each dimension are added together to form a cumulative time rate of change. Eqn. (3.1) is then written as

$$
\frac{\partial \mathbf{U}}{\partial t}=\mathcal{L}(\mathbf{U}) \equiv-\frac{\partial \mathbf{F}}{\partial x}
$$

A method of lines approach is then taken to integrate the system forward in time. Typically, SSP-RK methods of a suitable order from Shu and Osher [29] or Spiteri \& Ruuth [33], [34] are used for the time integration. For simplicity, the domain in the xdirection is covered with uniform zones, where the $j^{\text {th }}$ zone spans the interval $I_{j}=\left[x_{j-1 / 2}, x_{j+1 / 2}\right]$ with zone center $x_{j}=\left(x_{j-1 / 2}+x_{j+1 / 2}\right) / 2$ and zone size $\Delta x=\left(x_{j+1 / 2}-x_{j-1 / 2}\right)$. The update term in eqn. (3.2) can then be written as

$$
\mathcal{L}\left(\mathbf{U}_{j}(t)\right) \equiv-\frac{1}{\Delta x}\left(\hat{\mathbf{F}}_{j+1 / 2}-\hat{\mathbf{F}}_{j-1 / 2}\right)
$$


where $\hat{\mathbf{F}}_{j+1 / 2}$ is a suitably high order, properly upwinded, numerical flux defined at the zone boundary $j+1 / 2$.

Construction of properly upwinded numerical fluxes for finite difference WENO schemes is described in several sources including, in great detail, in section II of Balsara and Shu [1]. As a result, we only touch on it with the utmost brevity, describing the simplest case which is the construction of the LF flux. The numerical flux at a zone boundary, $j+1 / 2$, can, therefore, be split into a purely right-going flux and a purely left-going flux as follows

$\hat{\mathbf{F}}_{j+1 / 2}^{L F}=\frac{1}{2}\left(\mathbf{F}_{L}+\lambda \mathbf{U}_{L}\right)+\frac{1}{2}\left(\mathbf{F}_{R}-\lambda \mathbf{U}_{R}\right)$

Here $\lambda$ is at least slightly larger than the absolute value of the fastest speed going in either direction along the x-axis. The above equation is slightly impressioninstic and is intended to show the right-going part of the flux $\hat{\mathbf{F}}_{j+1 / 2}^{+} \equiv\left(\mathbf{F}_{L}+\lambda \mathbf{U}_{L}\right) / 2$, and the leftgoing part of the flux, $\hat{\mathbf{F}}_{j+1 / 2}^{-} \equiv\left(\mathbf{F}_{R}-\lambda \mathbf{U}_{R}\right) / 2$. In practice, $\hat{\mathbf{F}}_{j+1 / 2}^{+}$is obtained via a high order WENO reconstruction procedure that is applied to a set of stencils, all of which include the zone " $j$ ". For example, a fifth order WENO reconstruction would involve the zones $\{j-2, j-1, j, j+1, j+2\}$. In classical fifth order finite difference WENO, this is obtained by using the $r=3$ WENO reconstruction described in Sub-section II.1. Also, in practice, $\hat{\mathbf{F}}_{j+1 / 2}^{-}$is obtained via a high order WENO reconstruction procedure that is applied to a set of stencils, all of which include the zone " $j+1$ ". For example, a fifth order WENO reconstruction would involve the zones $\{j-1, j, j+1, j+2, j+3\}$.

\section{III.1) Fifth Order WENO Scheme with Adaptive Order - WENO-AO(5,3)}

It is now possible to illustrate the differences between classical finite difference WENO and finite difference WENO-AO of the same order. Interestingly, this also 
enables us to better understand the adaptive order WENO-AO schemes. We focus on the fifth order WENO-AO schemes in this sub-section. They are denoted by WENO-AO $(5,3)$. In classical fifth order finite difference WENO, the reconstruction of $\hat{\mathbf{F}}_{j+1 / 2}^{+}$uses $\mathrm{r}=3$ WENO reconstruction from Section II.1 applied to the zones $\{j-2, j-1, j, j+1, j+2\}$. In the adaptive order WENO-AO(5,3) reconstruction, we work with the same set of zones to obtain $\hat{\mathbf{F}}_{j+1 / 2}^{+}$. However, this is obtained by a non-linear hybridization between the centered stencil $S_{3}^{r 5}$ which is centered at zone " $j$ " along with the three $r=3$ CWENO stencils $S_{1}^{r 3}, S_{2}^{r 3}$ and $S_{3}^{r 3}$ that are also include zone " $j$ ”. Notice that classical WENO will achieve fifth order accuracy only if the large stencil carries a smooth solution. Otherwise, the solution will graciously reduce in order to third order because the smoothness measures permit us to pick out the smoothest stencil amongst the stencils $S_{1}^{r 3}, S_{2}^{r 3}$ and $\mathrm{S}_{3}^{r 3}$. Now realize that WENO-AO(5,3) should do something that is quite analogous. This is because it is non-linearly hybridized between the fifth order central stencil $S_{3}^{r 5}$ and the three third order $\mathrm{r}=3$ CWENO stencils $\mathrm{S}_{1}^{r 3}, \mathrm{~S}_{2}^{r 3}$ and $\mathrm{S}_{3}^{r 3}$. As a result, WENO-AO $(5,3)$ will also have an order of accuracy that is at best fifth order but can drop to third order if the larger stencil is non-smooth. Also realize that prior pactitioners have found that the $r=3$ CWENO reconstruction is extraordinarily stable with the result that the WENO$\mathrm{AO}(5,3)$ is guaranteed to be at least stable at third order along with having an ability to pick out extrema.

The details of this non-linear hybridization for WENO-AO(5,3) will be described in the next paragraph. However, it is very important to mention that the intuitive underpinnings of this method derive from the very recent, and very nice, paper by Zhu and Qiu [37], who hybridized the fifth order central stencil $S_{3}^{r 5}$ and a Van Albada-type of limiter. The Van Albada limiter is a TVD-class limiter which can clip extrema. For that reason, it is more advisable to nonlinearly hybridize the scheme with a suitable $r=3$ CWENO scheme as is done here. Of course, when the non-linear hybridization does not invoke the lower order scheme, both the finite difference $\operatorname{WENO}-\mathrm{AO}(5,3)$ scheme 
described here and the WENO-ZQ scheme described in Zhu and Qiu [37] achieve fifth order accuracy.

Now let us focus on a detailed description of WENO-AO(5,3). This description is important because we will later on show that it will also open the door to other families of WENO-AO schemes. The method is described by two parameters, $\gamma_{H i}$ and $\gamma_{L o}$, both of which are always less than unity. The linear weights for the stencils $\mathrm{S}_{3}^{r 5}$ and the stencils $\mathrm{S}_{1}^{r 3}, \mathrm{~S}_{2}^{r 3}$ and $\mathrm{S}_{3}^{r 3}$ are given by

$\gamma_{3}^{r 5}=\gamma_{H i} \quad ; \quad \gamma_{1}^{r 3}=\left(1-\gamma_{H i}\right)\left(1-\gamma_{L o}\right) / 2 \quad ; \quad \gamma_{2}^{r 3}=\left(1-\gamma_{H i}\right) \gamma_{L o} \quad ; \quad \gamma_{3}^{r 3}=\left(1-\gamma_{H i}\right)\left(1-\gamma_{L o}\right) / 2$

Notice that $\mathrm{S}_{2}^{r 3}$ is the central stencil of the $\mathrm{r}=3 \mathrm{CWENO}$ reconstruction, so it should carry a higher linear weight than the other two stencils of the $r=3$ CWENO reconstruction; this helps to make the CWENO centrally biased. Also notice that $\gamma_{1}^{r 3}+\gamma_{2}^{r 3}+\gamma_{3}^{r 3}=1-\gamma_{H i}$. Typically, we set $\gamma_{H i} \in[0.85,0.95]$ and $\gamma_{L o} \in[0.85,0.95]$. These numbers themselves give us a glimpse of what is afoot. When a suitable comparison of the smoothness indicators shows that the large central stencil $\mathrm{S}_{3}^{r 5}$ is smooth we want most (or all) of our reconstruction to come from the large central stencil. However, when a suitable comparison of the smoothness indicators shows that the large central stencil is nonsmooth, we want most (or all) of our reconstruction to be weighted towards our very stable, third order accurate, extrema-preserving $r=3 \mathrm{CWENO}$ reconstruction. In the next paragraph we describe the construction of the non-linear weights. In the paragraph after that, we describe the assembly of the non-linearly hybridized higher order reconstruction.

We now describe the process of obtaining the non-linear weights for WENO$\mathrm{AO}(5,3)$ reconstruction. To avoid loss of order at inflection points we use the smoothness indicators to define 


$$
\tau=\frac{1}{3}\left(\left|\beta_{3}^{r 5}-\beta_{1}^{r 3}\right|+\left|\beta_{3}^{r 5}-\beta_{2}^{r 3}\right|+\left|\beta_{3}^{r 5}-\beta_{3}^{r 3}\right|\right)
$$

Using the smoothness indicators again, and following Borges et al. [3], we can obtain the un-normalized weights as

$$
\begin{array}{ll}
w_{3}^{r 5}=\gamma_{3}^{r 5}\left(1+\tau^{2} /\left(\beta_{3}^{r 5}+\varepsilon\right)^{2}\right) \quad ; \quad w_{1}^{r 3}=\gamma_{1}^{r 3}\left(1+\tau^{2} /\left(\beta_{1}^{r 3}+\varepsilon\right)^{2}\right) ; \\
w_{2}^{r 3}=\gamma_{2}^{r 3}\left(1+\tau^{2} /\left(\beta_{2}^{r 3}+\varepsilon\right)^{2}\right) \quad ; \quad w_{3}^{r 3}=\gamma_{3}^{r 3}\left(1+\tau^{2} /\left(\beta_{3}^{r 3}+\varepsilon\right)^{2}\right)
\end{array}
$$

If the solution is not dominated by inflection points, it may even be acceptable to use the original WENO strategy for obtaining the un-normalized weights given by

$$
\begin{aligned}
& w_{3}^{r 5}=\gamma_{3}^{r 5} /\left(\beta_{3}^{r 5}+\varepsilon\right)^{2} ; w_{1}^{r 3}=\gamma_{1}^{r 3} /\left(\beta_{1}^{r 3}+\varepsilon\right)^{2} ; \\
& w_{2}^{r 3}=\gamma_{2}^{r 3} /\left(\beta_{2}^{r 3}+\varepsilon\right)^{2} ; w_{3}^{r 3}=\gamma_{3}^{r 3} /\left(\beta_{3}^{r 3}+\varepsilon\right)^{2}
\end{aligned}
$$

Here $\varepsilon$ is a very tiny number, typically $\varepsilon \square 10^{-12}$. We have found eqn. (3.7b) to be a more stable option while eqn. (3.7a) is a more accurate option. In practice, we have used eqn. (3.7a) in this paper. The normalized weights are given by

$$
\begin{array}{lll}
\bar{w}_{3}^{r 5}=w_{3}^{r 5} /\left(w_{3}^{r 5}+w_{1}^{r 3}+w_{2}^{r 3}+w_{3}^{r 3}\right) & ; \quad \bar{w}_{1}^{r 3}=w_{1}^{r 3} /\left(w_{3}^{r 5}+w_{1}^{r 3}+w_{2}^{r 3}+w_{3}^{r 3}\right) ; \\
\bar{w}_{2}^{r 3}=w_{2}^{r 3} /\left(w_{3}^{r 5}+w_{1}^{r 3}+w_{2}^{r 3}+w_{3}^{r 3}\right) & ; \quad \bar{w}_{3}^{r 3}=w_{3}^{r 3} /\left(w_{3}^{r 5}+w_{1}^{r 3}+w_{2}^{r 3}+w_{3}^{r 3}\right)
\end{array}
$$

This completes the description of the normalized, non-linear weights for WENO-AO $(5,3)$.

Say we denote the reconstructed polynomial from $\operatorname{WENO}-\mathrm{AO}(5,3)$ as $\mathrm{P}^{\mathrm{AO}(5,3)}(x)$. Our task in this paragraph is to describe the construction of the order-preserving, nonlinearly hybridized, fifth order polynomial $\mathrm{P}^{\mathrm{AO}(5,3)}(x)$. The non-linear weights should be combined in such a way that when all the smoothness indicators seem to have almost 
similar values then only the higher order scheme is obtained. Such a combination strategy was demonstrated in Zhu and Qiu [37] for non-linearly hybridizing a fifth order polynomial with the two linear polynomials that make up the Van Albada limiter. A similar idea can be used to make a non-linear hybridization between the fifth order polynomial associated with the central $S_{3}^{r 5}$ stencil and the three third order polynomials for the stencils, $S_{1}^{r 3}, S_{2}^{r 3}$ and $S_{3}^{r 3}$. The latter three stencils are associated with the $r=3$ CWENO reconstruction. Realize, therefore, that when the four smoothness measures associated with these four stencils have closely similar values, we have $\bar{w}_{3}^{r 5} \rightarrow \gamma_{3}^{r 5}$, $\bar{w}_{1}^{r 3} \rightarrow \gamma_{1}^{r 3}, \bar{w}_{2}^{r 3} \rightarrow \gamma_{2}^{r 3}$ and $\bar{w}_{3}^{r 3} \rightarrow \gamma_{3}^{r 3}$. We then require that when the limits specified by the previous sentence are attained, we have $\mathrm{P}^{\mathrm{AO}(5,3)}(x) \rightarrow \mathrm{P}_{3}^{r 5}(x)$. This is achieved by the following definition

$$
\begin{aligned}
\mathrm{P}^{\mathrm{AO}(5,3)}(x) & =\frac{\bar{w}_{3}^{r 5}}{\gamma_{3}^{r 5}}\left(\mathrm{P}_{3}^{r 5}(x)-\gamma_{1}^{r 3} \mathrm{P}_{1}^{r 3}(x)-\gamma_{2}^{r 3} \mathrm{P}_{2}^{r 3}(x)-\gamma_{3}^{r 3} \mathrm{P}_{3}^{r 3}(x)\right) \\
& +\bar{w}_{1}^{r 3} \mathrm{P}_{1}^{r 3}(x)+\bar{w}_{2}^{r 3} \mathrm{P}_{2}^{r 3}(x)+\bar{w}_{3}^{r 3} \mathrm{P}_{3}^{r 3}(x)
\end{aligned}
$$

Notice that in the limit where the smoothness indicators for the $\mathrm{S}_{3}^{r 5}, \mathrm{~S}_{1}^{r 3}, \mathrm{~S}_{2}^{r 3}$ and $\mathrm{S}_{3}^{r 3}$ stencils all have closely similar values, we do have $\mathrm{P}^{\mathrm{AO}(5,3)}(x) \rightarrow \mathrm{P}_{3}^{r 5}(x)$. In the limit where the larger stencil has a very non-smooth solution, we have $\bar{w}_{3}^{r 5} \square \bar{w}_{1}^{r 3}$ or $\bar{w}_{3}^{r 5} \square \bar{w}_{2}^{r 3}$ or $\bar{w}_{3}^{r 5} \square \bar{w}_{3}^{r 3}$. This ensures that the smoothest of the $r=3$ CWENO stencils will be sought out by the reconstruction polynomial. Notice that the non-linear hybridization that we sought at the beginning of this paragraph has been found via eqn. (3.9).

Some implementation details are given here to make it easy for implementers to implement the WENO-AO(5,3) reconstruction scheme that we have described here. Recall that we always work in terms of the coefficients of the Legendre basis. In this paragraph we make explicit the process of obtaining $\mathrm{P}^{\mathrm{AO}(5,3)}(x)$ for the " 0 " zone, since 
Section II was defined entirely in terms of that zone. Of course, the zones can be shifted so that the reconstruction can be obtained in any zone. The implementation is described via the following steps:-

Step 1) Let $\mathrm{u}_{x}^{r 3 ; 1}$ and $\mathrm{u}_{x 2}^{r 3 ; 1}$ be the coefficients of $\mathrm{P}_{1}^{r 3}(x)$; they are obtained using eqn. (2.3). Let $\mathrm{u}_{x}^{r 3 ; 2}$ and $\mathrm{u}_{x 2}^{r 3 ; 2}$ be the coefficients of $\mathrm{P}_{2}^{r 3}(x)$; they are obtained using eqn. (2.4). Let $u_{x}^{r 3 ; 3}$ and $u_{x 2}^{r 3 ; 3}$ be the coefficients of $P_{3}^{r 3}(x)$; they are obtained using eqn. (2.5). The smoothness indicators for these three $r=3$ polynomials can be obtained using eqn. (2.6). Step 2) Let $\mathrm{u}_{x}^{r 5 ; 3}, \mathrm{u}_{x 2}^{r 5 ; 3}, \mathrm{u}_{x 3}^{r 5 ; 3}$ and $\mathrm{u}_{x 4}^{r 5 ; 3}$ be the coefficients of the central, fifth order polynomial $\mathrm{P}_{3}^{r 5}(x)$; they are obtained using eqn. (2.16). The smoothess indicator for this fifth order polynomial can be obtained using eqn. (2.19).

Step 3) Eqns. (3.6), (3.7) and (3.8) are evaluated in sequence and they give us the normalized non-linear weights $\bar{w}_{3}^{r 5}, \bar{w}_{1}^{r 3}, \bar{w}_{2}^{r 3}$ and $\bar{w}_{3}^{r 3}$.

Step 4) The coefficients of the polynomial described in eqn. (3.9) can now be explicitly written as

$$
\begin{aligned}
& \mathrm{u}_{x}^{\mathrm{AO}(5,3)}=\frac{\bar{w}_{3}^{r 5}}{\gamma_{3}^{r 5}}\left(\mathrm{u}_{x}^{r 5 ; 3}-\gamma_{1}^{r 3} \mathrm{u}_{x}^{r 3 ; 1}-\gamma_{2}^{r 3} \mathrm{u}_{x}^{r 3 ; 2}-\gamma_{3}^{r 3} \mathrm{u}_{x}^{r 3 ; 3}\right)+\bar{w}_{1}^{r 3} \mathrm{u}_{x}^{r 3 ; 1}+\bar{w}_{2}^{r 3} \mathrm{u}_{x}^{r 3 ; 2}+\bar{w}_{3}^{r 3} \mathrm{u}_{x}^{r 3 ; 3} ; \\
& \mathrm{u}_{x 2}^{\mathrm{AO}(5,3)}=\frac{\bar{w}_{3}^{r 5}}{\gamma_{3}^{r 5}}\left(\mathrm{u}_{x 2}^{r 5 ; 3}-\gamma_{1}^{r 3} \mathrm{u}_{x 2}^{r 3 ; 1}-\gamma_{2}^{r 3} \mathrm{u}_{x 2}^{r 3 ; 2}-\gamma_{3}^{r 3} \mathrm{u}_{x 2}^{r 3 ; 3}\right)+\bar{w}_{1}^{r 3} \mathrm{u}_{x 2}^{r 3 ; 1}+\bar{w}_{2}^{r 3} \mathrm{u}_{x 2}^{r 3 ; 2}+\bar{w}_{3}^{r 3} \mathrm{u}_{x 2}^{r 3 ; 3} ; \\
& \mathrm{u}_{x 3}^{\mathrm{AO}(5,3)}=\frac{\bar{w}_{3}^{r 5}}{\gamma_{3}^{r 5}} \mathrm{u}_{x 3}^{r 5 ; 3} ; \mathrm{u}_{x 4}^{\mathrm{AO}(5,3)}=\frac{\bar{w}_{3}^{r 5}}{\gamma_{3}^{r 5}} \mathrm{u}_{x 4}^{r 5 ; 3}
\end{aligned}
$$

Step 5) The final WENO-AO(5,3) reconstructed polynomial is obtained by using eqn. (2.13) and is explicitly written as

$$
\mathrm{P}^{\mathrm{AO}(5,3)}(x)=\mathrm{u}_{0}+\mathrm{u}_{x}^{\mathrm{AO}(5,3)} \mathrm{L}_{1}(x)+\mathrm{u}_{x 2}^{\mathrm{AO}(5,3)} \mathrm{L}_{2}(x)+\mathrm{u}_{x 3}^{\mathrm{AO}(5,3)} \mathrm{L}_{3}(x)+\mathrm{u}_{x 4}^{\mathrm{AO}(5,3)} \mathrm{L}_{4}(x)
$$

Eqns. (3.10) and (3.11), along with the narrative in this paragraph show us that WENO$\mathrm{AO}(5,3)$ schemes are very easy to implement. This same ease of implementation extends 
to the other WENO-AO reconstruction schemes described in subsequent sub-sections. All the steps can be concatenated into the body a single loop that loops over the different zones. This makes the implementation very cache-friendly since most of the variables in the loop can reside in the cache of the processor. Similarly, the algorithm is very friendly to implementation on GPUs.

Let us try to understand eqns. (3.10) and (3.11) at an intuitive level. Eqn. (3.10) shows us that when $\bar{w}_{3}^{r 5} \rightarrow \gamma_{3}^{r 5}$ we have $\mathrm{u}_{x}^{\mathrm{AO}(5,3)} \rightarrow \mathrm{u}_{x}^{r 5 ; 3}, \mathrm{u}_{x 2}^{\mathrm{AO}(5,3)} \rightarrow \mathrm{u}_{x 2}^{r 5 ; 3}, \mathrm{u}_{x 3}^{\mathrm{AO}(5,3)} \rightarrow \mathrm{u}_{x 3}^{r 5 ; 3}$ and $\mathrm{u}_{x 4}^{\mathrm{AO}(5,3)} \rightarrow \mathrm{u}_{x 4}^{r 5 ; 3}$; so that $\mathrm{P}^{\mathrm{AO}(5,3)}(x) \rightarrow \mathrm{P}_{3}^{r 5}(x)$. However, when $\bar{w}_{3}^{r 5} \square \gamma_{3}^{r 5}$, we see that $\mathrm{u}_{x}^{\mathrm{AO}(5,3)}$ and $\mathrm{u}_{x 2}^{\mathrm{AO}(5,3)}$ tend towards their smoothest values from the $\mathrm{r}=3$ CWENO reconstruction. Furthermore, we see that when $\bar{w}_{3}^{r 5} \square \gamma_{3}^{r 5}$ we have $\mathrm{u}_{x 3}^{\mathrm{AO}(5,3)} \rightarrow 0$ and $\mathrm{u}_{x 4}^{\mathrm{AO}(5,3)} \rightarrow 0$. We see, therefore, that when the large central stencil $\mathrm{S}_{3}^{r 5}$ carries a nonsmooth solution, the cubic and quartic terms are very strongly suppressed by WENO$\mathrm{AO}(5,3)$. These cubic and quartic terms are most likely to need a monotonicity preserving treatment if they are not effectively suppressed when the solution is non-smooth. Since WENO-AO(5,3) suppresses these terms very effectively, it does not need the additional stabilization from a monotonicity preserving formulation. This same advantage extends to the higher order WENO-AO schemes described in subsequent sub-sections.

\section{III.2) Seventh Order WENO Scheme with Adaptive Order - WENO-AO(7,3)}

We focus on the seventh order WENO-AO scheme in this sub-section. It is denoted by WENO-AO(7,3). It is obtained by a non-linear hybridization between the centered stencil $\mathrm{S}_{4}^{r 7}$ which is centered at zone " $\mathrm{j}$ " along with the three $\mathrm{r}=3 \mathrm{CWENO}$ stencils $\mathrm{S}_{1}^{r 3}, \mathrm{~S}_{2}^{r 3}$ and $\mathrm{S}_{3}^{r 3}$ that are also include zone " $j$ ". In other words, the WENO$\mathrm{AO}(7,3)$ reconstruction is hybridized between the seventh order central stencil $\mathrm{S}_{4}^{r 7}$ and the three third order $r=3$ CWENO stencils $S_{1}^{r 3}, S_{2}^{r 3}$ and $S_{3}^{r 3}$. As a result, WENO-AO $(7,3)$ will also have an order of accuracy that is at best seventh order but can drop to third order 
if the larger stencil is non-smooth. We denote the reconstructed polynomial from WENO$\mathrm{AO}(7,3)$ as $\mathrm{P}^{\mathrm{AO}(7,3)}(x)$.

The purpose of this brief section is to highlight the analogies between the WENO$\mathrm{AO}(7,3)$ reconstruction and the WENO-AO $(5,3)$ reconstruction from the previous subsection. The method is still described by two parameters, $\gamma_{H i}$ and $\gamma_{L o}$, both of which are always less than unity. The linear weights are determined by expressions that are entirely analogous to eqn. (3.5). The linear weights for the stencils $S_{4}^{r 7}$ and the stencils $S_{1}^{r 3}, S_{2}^{r 3}$ and $S_{3}^{r 3}$ are given by

$\gamma_{4}^{r 7}=\gamma_{H i} \quad ; \quad \gamma_{1}^{r 3}=\left(1-\gamma_{H i}\right)\left(1-\gamma_{L o}\right) / 2 \quad ; \quad \gamma_{2}^{r 3}=\left(1-\gamma_{H i}\right) \gamma_{L o} \quad ; \quad \gamma_{3}^{r 3}=\left(1-\gamma_{H i}\right)\left(1-\gamma_{L o}\right) / 2$

The analogue of eqn. (3.6) becomes

$\tau=\frac{1}{3}\left(\left|\beta_{4}^{r 7}-\beta_{1}^{r 3}\right|+\left|\beta_{4}^{r 7}-\beta_{2}^{r 3}\right|+\left|\beta_{4}^{r 7}-\beta_{3}^{r 3}\right|\right)$

Using the smoothness indicators, the analogue of eqn. (3.7a) for the un-normalized weights becomes

$$
\begin{array}{ll}
w_{4}^{r 7}=\gamma_{4}^{r 7}\left(1+\tau^{2} /\left(\beta_{4}^{r 7}+\varepsilon\right)^{2}\right) ; & w_{1}^{r 3}=\gamma_{1}^{r 3}\left(1+\tau^{2} /\left(\beta_{1}^{r 3}+\varepsilon\right)^{2}\right) ; \\
w_{2}^{r 3}=\gamma_{2}^{r 3}\left(1+\tau^{2} /\left(\beta_{2}^{r 3}+\varepsilon\right)^{2}\right) ; & w_{3}^{r 3}=\gamma_{3}^{r 3}\left(1+\tau^{2} /\left(\beta_{3}^{r 3}+\varepsilon\right)^{2}\right)
\end{array}
$$

The analogue of eqn. (3.7b) for the un-normalized weights becomes

$$
\begin{aligned}
& w_{4}^{r 7}=\gamma_{4}^{r 7} /\left(\beta_{4}^{r 7}+\varepsilon\right)^{2} ; w_{1}^{r 3}=\gamma_{1}^{r 3} /\left(\beta_{1}^{r 3}+\varepsilon\right)^{2} ; \\
& w_{2}^{r 3}=\gamma_{2}^{r 3} /\left(\beta_{2}^{r 3}+\varepsilon\right)^{2} ; w_{3}^{r 3}=\gamma_{3}^{r 3} /\left(\beta_{3}^{r 3}+\varepsilon\right)^{2}
\end{aligned}
$$


In practice, we have used eqn. (3.14a) in this paper. An equation that is closely analogous to eqn. (3.8) then gives the normalized, non-linear weights as

$$
\begin{array}{lll}
\bar{w}_{4}^{r 7}=w_{4}^{r 7} /\left(w_{4}^{r 7}+w_{1}^{r 3}+w_{2}^{r 3}+w_{3}^{r 3}\right) & ; \quad \bar{w}_{1}^{r 3}=w_{1}^{r 3} /\left(w_{4}^{r 7}+w_{1}^{r 3}+w_{2}^{r 3}+w_{3}^{r 3}\right) ; \\
\bar{w}_{2}^{r 3}=w_{2}^{r 3} /\left(w_{4}^{r 7}+w_{1}^{r 3}+w_{2}^{r 3}+w_{3}^{r 3}\right) & ; \quad \bar{w}_{3}^{r 3}=w_{3}^{r 3} /\left(w_{4}^{r 7}+w_{1}^{r 3}+w_{2}^{r 3}+w_{3}^{r 3}\right)
\end{array}
$$

The reconstructed polynomial $\mathrm{P}^{\mathrm{AO}(7,3)}(x)$ is then given by an expression that is very analogous to eqn. (3.9) and is given by

$$
\begin{aligned}
\mathrm{P}^{\mathrm{AO}(7,3)}(x) & =\frac{\bar{w}_{4}^{r 7}}{\gamma_{4}^{r 7}}\left(\mathrm{P}_{4}^{r 7}(x)-\gamma_{1}^{r 3} \mathrm{P}_{1}^{r 3}(x)-\gamma_{2}^{r 3} \mathrm{P}_{2}^{r 3}(x)-\gamma_{3}^{r 3} \mathrm{P}_{3}^{r 3}(x)\right) \\
& +\bar{w}_{1}^{r 3} \mathrm{P}_{1}^{r 3}(x)+\bar{w}_{2}^{r 3} \mathrm{P}_{2}^{r 3}(x)+\bar{w}_{3}^{r 3} \mathrm{P}_{3}^{r 3}(x)
\end{aligned}
$$

This completes our description of WENO-AO(7,3). We have implemented such a scheme and for idealized test problems and it does indeed achieve seventh order of accuracy. As in the previous section, the scheme does not need any monotonicity preserving fix.

\section{III.3) Formally Ninth Order WENO Schemes with Adaptive Order - WENO-AO(9,3)}

It may always be conjectured that the game-plan from the previous two sections can be extended to obtain a formally ninth order WENO-AO scheme. Indeed, as a matter of formal definition, this can be done. The scheme is denoted by WENO-AO(9,3). It is obtained by a non-linear hybridization between the centered stencil $\mathrm{S}_{5}^{r 9}$ which is centered at zone " $\mathrm{j}$ " along with the three $\mathrm{r}=3$ CWENO stencils $\mathrm{S}_{1}^{r 3}, \mathrm{~S}_{2}^{r 3}$ and $\mathrm{S}_{3}^{\mathrm{r} 3}$ that are also include zone "j". Having seen the development in the previous section, it is easy to see that any entity " $A$ " that has the formal structure $A_{4}^{r 7}$ should now be replaced by $A_{5}^{r 9}$. In other words, making the replacement from the previous sentence in sub-Section III.2 will give us a WENO-AO $(9,3)$ reconstruction strategy. We have constructed such a scheme and it does achieve ninth order of accuracy on certain idealized problems. 


\section{III.4) A Recursively Defined Seventh Order WENO Scheme with Adaptive Order - WENO-AO(7,5,3)}

Our experience shows that WENO-AO(7,3) is a useful and well-behaved scheme. However, a purist might still detect a deficiency in it. Notice that when the central stencil $\mathrm{S}_{4}^{r 7}$ has a non-smooth solution, the WENO-AO $(7,3)$ will graciously degenerate to a third order scheme. However, it is always possible that the stencil $\mathrm{S}_{3}^{r 5}$ has a smooth solution even when the solution on the stencil $\mathrm{S}_{4}^{r 7}$ is non-smooth. It is, therefore, possible to recursively hybridize between the potentially seventh order accurate polynomial $\mathrm{P}^{\mathrm{AO}(7,3)}(x)$ and the potentially fifth order accurate polynomial $\mathrm{P}^{\mathrm{AO}(5,3)}(x)$. As one might expect, this is done by examining the smoothness indicator $\beta_{4}^{r 7}$ corresponding to the stencil $\mathrm{S}_{4}^{r 7}$ and the smoothness indicator $\beta_{3}^{r 5}$ corresponding to the stencil $\mathrm{S}_{3}^{r 5}$. We define the two un-normalized weights given by

$v^{\mathrm{AO}(7,3)}=\gamma_{H i}\left(1+\sigma /\left(\beta_{4}^{r 7}+\varepsilon\right)\right) ; \quad v^{\mathrm{AO}(5,3)}=\left(1-\gamma_{H i}\right)\left(1+\sigma /\left(\beta_{3}^{r 5}+\varepsilon\right)\right)$

with $\sigma \equiv\left|\beta_{4}^{r 7}-\beta_{3}^{r 5}\right|$

The normalized, non-linear weights are given by

$\bar{v}^{\mathrm{AO}(7,3)}=v^{\mathrm{AO}(7,3)} /\left(v^{\mathrm{AO}(7,3)}+v^{\mathrm{AO}(5,3)}\right) \quad ; \quad \bar{v}^{\mathrm{AO}(5,3)}=v^{\mathrm{AO}(5,3)} /\left(v^{\mathrm{AO}(7,3)}+v^{\mathrm{AO}(5,3)}\right)$

The recursively hybridized WENO-AO(7,5,3) scheme will have a reconstructed polynomial which we denote by $\mathrm{P}^{\mathrm{AO}(7,5,3)}(x)$. The polynomial $\mathrm{P}^{\mathrm{AO}(7,5,3)}(x)$ is given by

$$
\mathrm{P}^{\mathrm{AO}(7,5,3)}(x)=\frac{\bar{v}^{\mathrm{AO}(7,3)}}{\gamma_{H i}}\left(\mathrm{P}^{\mathrm{AO}(7,3)}(x)-\left(1-\gamma_{H i}\right) \mathrm{P}^{\mathrm{AO}(5,3)}(x)\right)+\bar{v}^{\mathrm{AO}(5,3)} \mathrm{P}^{\mathrm{AO}(5,3)}(x)
$$


This completes our description of the recursive process for obtaining $\mathrm{P}^{\mathrm{AO}(7,5,3)}(x)$.

Notice that when the solution is smooth on the stencils $\mathrm{S}_{4}^{r 7}$ and $\mathrm{S}_{3}^{r 5}$ we hope to obtain $\bar{v}^{\mathrm{AO}(7,3)} \rightarrow \gamma_{H i}$ and $\bar{v}^{\mathrm{AO}(5,3)} \rightarrow\left(1-\gamma_{H i}\right)$. In that limit, it is easy to show that $\mathrm{P}^{\mathrm{AO}(7,5,3)}(x) \rightarrow \mathrm{P}^{\mathrm{AO}(7,3)}(x) \rightarrow \mathrm{P}_{4}^{r 7}(x)$. However, please also notice that each of the polynomials $\mathrm{P}^{\mathrm{AO}(7,3)}(x)$ and $\mathrm{P}^{\mathrm{AO}(5,3)}(x)$ are individually designed so that they are nonlinearly stable. Eqn. (3.17) should be compared to eqns. (3.7a) and (3.14a). The square in eqns. (3.7a) and (3.14a) ensures that we switch quite readily to the stabler and lower order stencil. The absence of a square in eqn. (3.17) ensures that we do not switch very readily from $\mathrm{P}^{\mathrm{AO}(7,3)}(x)$ to $\mathrm{P}^{\mathrm{AO}(5,3)}(x)$. This is a good choice because both $\mathrm{P}^{\mathrm{AO}(7,3)}(x)$ and $\mathrm{P}^{\mathrm{AO}(5,3)}(x)$ are individually designed to be stable. We can always encounter the very unlikely circumstance where the smoothness indicators $\beta_{4}^{r 7}$ and $\beta_{3}^{r 5}$ have comparable values even though the stencils $\mathrm{S}_{4}^{r 7}$ and $\mathrm{S}_{3}^{r 5}$ both carry a non-smooth solution. In such a circumstance, $\mathrm{P}^{\mathrm{AO}(7,5,3)}(x)$ will still be a non-linearly stable polynomial that is made of a convex combination of two non-linearly stable polynomials, i.e., $\mathrm{P}^{\mathrm{AO}(7,3)}(x)$ and $\mathrm{P}^{\mathrm{AO}(5,3)}(x)$. This convexity property obtains as long as $\gamma_{H i} \bar{v}^{\mathrm{AO}(5,3)} \geq\left(1-\gamma_{H i}\right) \bar{v}^{\mathrm{AO}(7,3)}$. In most normal circumstances, we expect this property to be respected because it says that the ratio of the non-linear weight to the linear weight of the lower order stencil, i.e. $\bar{v}^{\mathrm{AO}(5,3)} /\left(1-\gamma_{H i}\right)$, is greater than the corresponding ratio for the higher order stencil, i.e. $\bar{v}^{\mathrm{AO}(7,3)} / \gamma_{H i}$. When the convexity property is violated, it is best to reset $\mathrm{P}^{\mathrm{AO}(7,5,3)}(x) \rightarrow \mathrm{P}^{\mathrm{AO}(7,3)}(x)$. Realistically speaking, we almost always expect $\beta_{4}^{r 7}>\beta_{3}^{r 5}$, so that we expect that the convexity property will almost never be violated. This completes our description of WENO-AO $(7,5,3)$ reconstruction which yields the polynomial $\mathrm{P}^{\mathrm{AO}(7,5,3)}(x)$.

Please note that the computer implementation of $\operatorname{WENO}-\mathrm{AO}(7,5,3)$ reconstruction is only slightly more expensive than the computer implementation of 
WENO-AO $(7,3)$. This is because one only has to pay an additional cost for the construction of the smoothness indicator $\beta_{3}^{r 5}$ and the coefficients of the polynomial $\mathrm{P}_{3}^{r 5}(x)$ that go towards its evaluation. We hope that the WENO-AO(7,5,3) plays an important role in turbulence simulations of compressible flow where it is important to have a stencil that is as close to a central stencil as possible. In such turbulence simulations it is very important to preserve phase accuracy of flow features as much as possible. The WENO-AO(7,5,3) scheme fulfils on that goal because it graciously transitions from a seventh order centered stencil to a fifth order centered stencil to a super-stable third order CWENO formulation that can robustly capture shocks as well as physical extrema.

It is possible to carry this recursive process further. Thus, non-linear hybridization between $\mathrm{P}^{\mathrm{AO}(9,3)}(x)$ and $\mathrm{P}^{\mathrm{AO}(7,5,3)}(x)$ would yield a WENO-AO(9,7,5,3) scheme. While this might seem like overkill, we have indeed implemented a scheme that consists of a non-linear hybridization between $\mathrm{P}^{\mathrm{AO}(9,3)}(x)$ and $\mathrm{P}^{\mathrm{AO}(5,3)}(x)$ resulting in a WENO$\mathrm{AO}(9,5,3)$ scheme and we have found it to work well.

We also note that there is a modest analogy between MOOD (Multidimensional Optimal Order Detection) schemes (Clain, Diot and Loubère [5], Diot, Clain and Loubère [8], Diot, Loubère and Clain [9], Dumbser et al. [11]) which can have arbitrary order of accuracy between some designed maximum and some designed minimum and the WENO-AO $(7,5,3)$ reconstruction defined here. Both methods try to retain higher order accuracy while being willing to relinquish high order accuracy when that is required for the sake of stability. The difference is that MOOD schemes attempt to do a posteriori limiting, which can sometimes cause them to evaluate the update of a zone multiple times in the course of a timestep. By contrast, the WENO-AO schemes rely on an a priori limiter, which makes the limiting much simpler - there is only one update per zone. Although this paper is focused on finite difference WENO-AO schemes, it is also possible to develop finite volume and Discontinuous Galerkin limiters that are based on the same adaptive order philosophy. We will do that in later publications. 


\section{IV) Accuracy Analysis}

Several one-dimensional and multidimensional tests are presented in this section to demonstrate the accuracy of our WENO-AO methods. All the WENO-AO schemes in this section and the next were run with $\gamma_{H i}=0.85$ and $\gamma_{L o}=0.85$. In order to have a fair comparison, the WENO-ZQ scheme by Zhu and Qiu [37] was always run with the large central stencil having a weight of 0.98 ; this is the authors' suggested value. We always used the LLF formulation for the fluxes in this paper, since it is not the goal of this paper to explore different basal Riemann solvers. We did not use any steepener algorithm for the simulations shown here; nor did we use any monotonicity preserving method. As a result, we are showing the native accuracy of the finite difference WENO-AO schemes.

The dispersion and dissipation accuracy of a higher order method is also of interest to people who simulate turbulence. While this is not traditionally viewed as accuracy analysis of a higher order scheme, it gives a measure of the phase accuracy of the method. For that reason, we provide a brief sub-section on dispersion and dissipation accuracy of WENO-AO schemes at the end of this Section.

\section{IV.a) Linear Advection of Sinusoidal Profiles}

We solve the scalar advection equation $\mathrm{u}_{t}+\mathrm{u}_{x}=0$ on the one-dimensional periodic domain $x \in[-1,1]$ with initial condition $\mathrm{u}_{0}(x)=\sin (\pi x)$. The problem was run to a final time of unity with a fourth order accurate Runge-Kutta timestepping scheme. The problem was run on the coarsest mesh with a CFL of 0.6 . If $\Omega$ is the optimal spatial accuracy of the scheme, the CFL on finer meshes was scaled as $(\Delta x)^{(\Omega / 4)}$ so that the true spatial accuracy of the method can be demonstrated. Realize, therefore, that the problem will be run on finer meshes with a CFL that is much smaller than 0.6 , thus raising the possibility of excessive error build-up on the finer meshes. Our experience shows, 
however, that this does not occur till rather fine meshes are reached, thereby enabling us to demonstrate the spatial order of accuracy of the scheme.

Table I shows the accuracy as a function of increasing number of zones for the WENO-ZQ scheme, the WENO-AO $(5,3)$ scheme, the WENO-AO $(7,5,3)$ scheme and the WENO-AO $(9,5,3)$ scheme. For this very smooth problem, the WENO-AO $(7,3)$ scheme has an accuracy that is effectively identical to the WENO-AO(7,5,3) scheme; therefore, we do not show it here. Likewise, the WENO-AO(9,3) scheme has an accuracy that is effectively identical to the WENO-AO $(9,5,3)$ scheme; which is why we do not show it here. We see that all the methods that are shown in Table I reach their design accuracies. We also see that the accuracy of our $\operatorname{WENO}-\operatorname{AO}(5,3)$ scheme is more than half an order of magnitude better than the accuracy of the WENO-ZQ scheme on all the meshes. This demonstrates that the non-linear hybridization with $\mathrm{r}=3 \mathrm{CWENO}$, while adding almost negligibly to the cost of the method, has resulted in a substantial improvement in the quality of the solution.

The accuracy of the WENO-ZQ scheme has been improved somewhat because the large central stencil was given a linear weight of 0.98 . Even then, the accuracy of the WENO-ZQ scheme remains inferior to the accuracy of our WENO-AO $(5,3)$ scheme. Our WENO-AO class of schemes are relatively unaffected by the value of $\gamma_{H i}$ and $\gamma_{L o}$. The accuracy of a robust scheme should be relatively independent of input parameters, so we view that as a strength of our WENO-AO schemes.

Table I shows the accuracy of the sine wave advection test with WENO-ZQ, WENOAO(5,3), WENO-AO(7,5,3) and WENO-AO(9,5,3) schemes

\begin{tabular}{|l|l|l|r|l|r|}
\hline Method & \# of Zones & L1 Error & L1 Accuracy & L $\infty$ Error & L $\infty$ Accuracy \\
\hline WENO-ZQ & & & & & \\
\hline & 10 & $1.3473 \mathrm{E}-02$ & & $2.9631 \mathrm{E}-02$ & \\
\hline & 20 & $6.9263 \mathrm{E}-04$ & 4.28 & $2.5886 \mathrm{E}-03$ & 3.52 \\
\hline
\end{tabular}




\begin{tabular}{|c|c|c|c|c|c|}
\hline & 40 & $5.0068 \mathrm{E}-05$ & 3.79 & $2.5319 \mathrm{E}-04$ & 3.35 \\
\hline & 80 & $9.9071 \mathrm{E}-07$ & 5.66 & 7.4381E-06 & 5.09 \\
\hline \multicolumn{6}{|l|}{$\begin{array}{l}\text { WENO- } \\
\mathrm{AO}(5,3)\end{array}$} \\
\hline & 10 & $3.6382 \mathrm{E}-03$ & & $5.5511 \mathrm{E}-03$ & \\
\hline & 20 & $1.1024 \mathrm{E}-04$ & 5.04 & $1.7339 \mathrm{E}-04$ & 5.00 \\
\hline & 40 & $3.4890 \mathrm{E}-06$ & 4.98 & $5.4838 \mathrm{E}-06$ & 4.98 \\
\hline & 80 & $1.0944 \mathrm{E}-07$ & 4.99 & 1.7193E-07 & 5.00 \\
\hline \multicolumn{6}{|l|}{$\begin{array}{l}\text { WENO- } \\
\mathrm{AO}(7,5,3)\end{array}$} \\
\hline & 10 & $7.7366 \mathrm{E}-04$ & & $1.1084 \mathrm{E}-03$ & \\
\hline & 20 & $2.6284 \mathrm{E}-06$ & 8.20 & $3.7126 \mathrm{E}-06$ & 8.22 \\
\hline & 40 & $1.8914 \mathrm{E}-08$ & 7.12 & $2.9030 \mathrm{E}-08$ & 7.00 \\
\hline & 80 & $1.4562 \mathrm{E}-10$ & 7.02 & $2.2752 \mathrm{E}-10$ & 7.00 \\
\hline \multicolumn{6}{|l|}{$\begin{array}{l}\text { WENO- } \\
\mathrm{AO}(9,5,3)\end{array}$} \\
\hline & 10 & $5.4788 \mathrm{E}-04$ & & $8.9372 \mathrm{E}-04$ & \\
\hline & 20 & $3.5088 \mathrm{E}-07$ & 10.61 & $6.0682 \mathrm{E}-07$ & 10.52 \\
\hline & 40 & $6.0744 \mathrm{E}-10$ & 9.17 & $1.0090 \mathrm{E}-09$ & 9.23 \\
\hline & 80 & $1.4070 \mathrm{E}-12$ & 8.75 & $2.4215 \mathrm{E}-12$ & 8.70 \\
\hline
\end{tabular}

\section{IV.b) Non-linear Burgers Equation in One Dimension}

We solve the non-linear Burgers equation $\mathrm{u}_{t}+\left(\mathrm{u}^{2} / 2\right)_{x}=0$ on the onedimensional periodic domain $x \in[-1,1]$ with initial condition $\mathrm{u}_{0}(x)=0.25+0.5 \sin (\pi x)$. The problem was run to a final time of $1 / \pi$; which corresponds to a time that is before the formation of the shock. We used a fourth order accurate Runge-Kutta timestepping scheme. The problem was run on the coarsest mesh with a CFL of 0.6 and the CFL number was scaled down on finer meshes, as previously described. The same four 
schemes as before were run and the results are shown in Table II. We see that all the methods that are shown in Table II reach their design accuracies. We also see that the accuracy of our WENO-AO $(5,3)$ scheme is somewhat better than the accuracy of the WENO-ZQ scheme on all the meshes.

Table II shows the accuracy of the Burgers equation test with WENO-ZQ, WENOAO(5,3), WENO-AO(7,5,3) and WENO-AO(9,5,3) schemes

\begin{tabular}{|c|c|c|c|c|c|}
\hline Method & \# of Zones & L1 Error & L1 Accuracy & L $\infty$ Error & Lœ Accuracy \\
\hline \multicolumn{6}{|c|}{ WENO-ZQ } \\
\hline & 10 & 5.8154E-03 & & $1.9515 \mathrm{E}-02$ & \\
\hline & 20 & $7.4220 \mathrm{E}-04$ & 2.97 & $9.0760 \mathrm{E}-04$ & 4.43 \\
\hline & 40 & $3.6775 \mathrm{E}-05$ & 4.34 & $3.5689 \mathrm{E}-04$ & 1.35 \\
\hline & 80 & 8.9123E-07 & 5.37 & $1.2158 \mathrm{E}-05$ & 4.88 \\
\hline \multicolumn{6}{|l|}{$\begin{array}{l}\text { WENO- } \\
\mathrm{AO}(5,3)\end{array}$} \\
\hline & 10 & 4.9421E-03 & & $1.7277 \mathrm{E}-02$ & \\
\hline & 20 & $6.9353 \mathrm{E}-04$ & 2.83 & 4.8916E-03 & 1.82 \\
\hline & 40 & $2.9831 \mathrm{E}-05$ & 4.54 & $3.5742 \mathrm{E}-04$ & 3.77 \\
\hline & 80 & $8.5024 \mathrm{E}-07$ & 5.13 & $1.2158 \mathrm{E}-05$ & 4.88 \\
\hline \multicolumn{6}{|l|}{$\begin{array}{l}\text { WENO- } \\
\mathrm{AO}(7,5,3)\end{array}$} \\
\hline & 10 & 5.1209E-03 & & 1.9869E-02 & \\
\hline & 20 & 4.3334E-04 & 3.56 & $3.1234 \mathrm{E}-03$ & 2.67 \\
\hline & 40 & $1.1647 \mathrm{E}-05$ & 5.22 & 1.2882E-04 & 4.60 \\
\hline & 80 & $1.1629 \mathrm{E}-07$ & 6.65 & $1.7216 \mathrm{E}-06$ & 6.23 \\
\hline & 160 & $7.3990 \mathrm{E}-10$ & 7.30 & $1.3675 \mathrm{E}-08$ & 6.98 \\
\hline \multicolumn{6}{|l|}{$\begin{array}{l}\text { WENO- } \\
\mathrm{AO}(9,5,3)\end{array}$} \\
\hline & 10 & $5.5365 \mathrm{E}-03$ & & $2.0517 \mathrm{E}-02$ & \\
\hline
\end{tabular}




\begin{tabular}{|l|l|r|r|r|r|}
\hline & 20 & $5.7010 \mathrm{E}-04$ & 3.28 & $4.5932 \mathrm{E}-03$ & 2.16 \\
\hline & 40 & $7.0119 \mathrm{E}-06$ & 6.35 & $6.7292 \mathrm{E}-05$ & 6.09 \\
\hline & 80 & $2.9824 \mathrm{E}-08$ & 7.88 & $4.6522 \mathrm{E}-07$ & 7.18 \\
\hline & 160 & $6.3850 \mathrm{E}-11$ & 8.87 & $1.2238 \mathrm{E}-09$ & 8.57 \\
\hline
\end{tabular}

\section{IV.c) Euler Equations in Two Dimensions}

We solve the two-dimensional Euler equations on the two-dimensional periodic domain $(x, y) \in[0,2 \pi] \times[0,2 \pi]$. The pressure, $\mathrm{x}$-velocity and y-velocity were all set to unity. The ratio of specific heats was set to 1.4. The initial density was given by $\rho_{0}(x, y)=1+0.2 \sin (x+y)$ and the problem was run to a final time of 2 units with a fourth order accurate Runge-Kutta timestepping scheme. The problem was run on the coarsest mesh with a CFL of 0.3 and the CFL number was scaled down on finer meshes, as previously described. The same four schemes as before were run and the results are shown in Table III. We see that all the methods that are shown in Table III reach their design accuracies. (Since we used $\varepsilon=10^{-12}$, the accuracy of the ninth order scheme tapers off on the finest mesh.) We also see that the accuracy of our WENO-AO $(5,3)$ scheme is much better than the accuracy of the WENO-ZQ scheme on all the meshes.

Table III shows the accuracy of the two-dimensional Euler equation test with WENO-ZQ, WENO-AO(5,3), WENO-AO(7,5,3) and WENO-AO(9,5,3) schemes

\begin{tabular}{|l|l|l|r|r|r|}
\hline Method & \# of Zones & L1 Error & L1 Accuracy & L $\infty$ Error & L $\infty$ Accuracy \\
\hline WENO-ZQ & & & & & \\
\hline & $10 \times 10$ & $1.1627 \mathrm{E}-03$ & & $3.1556 \mathrm{E}-03$ & \\
\hline & $20 \times 20$ & $4.5302 \mathrm{E}-05$ & 4.68 & $2.0978 \mathrm{E}-04$ & 3.91 \\
\hline & $40 \times 40$ & $3.6967 \mathrm{E}-06$ & 3.62 & $2.1586 \mathrm{E}-05$ & 3.28 \\
\hline & $80 \times 80$ & $6.5304 \mathrm{E}-08$ & 5.82 & $8.2153 \mathrm{E}-07$ & 4.72 \\
\hline WENO- & & & & & \\
\hline
\end{tabular}




\begin{tabular}{|c|c|c|c|c|c|}
\hline $\mathrm{AO}(5,3)$ & & & & & \\
\hline & $10 \times 10$ & 8.9540E-04 & & $1.3543 \mathrm{E}-03$ & \\
\hline & $20 \times 20$ & $2.8275 \mathrm{E}-05$ & 4.98 & 4.4273E-05 & 4.93 \\
\hline & $40 \times 40$ & 8.8957E-07 & 4.99 & $1.3981 \mathrm{E}-06$ & 4.98 \\
\hline & $80 \times 80$ & $2.7885 \mathrm{E}-08$ & 5.00 & 4.3779E-08 & 5.00 \\
\hline WENO- & & & & & \\
\hline $\mathrm{AO}(7,5,3)$ & & & & & \\
\hline & $10 \times 10$ & $1.1253 \mathrm{E}-04$ & & $1.6059 \mathrm{E}-04$ & \\
\hline & $20 \times 20$ & $6.6656 \mathrm{E}-07$ & 7.40 & 9.4772E-07 & 7.40 \\
\hline & $40 \times 40$ & 4.8194E-09 & 7.11 & 7.3960E-09 & 7.00 \\
\hline & $80 \times 80$ & $3.7075 \mathrm{E}-11$ & 7.02 & $5.8410 \mathrm{E}-11$ & 6.98 \\
\hline WENO- & & & & & \\
\hline $\mathrm{AO}(9,5,3)$ & & & & & \\
\hline & $10 \times 10$ & 4.7889E-05 & & $1.0610 \mathrm{E}-04$ & \\
\hline & $20 \times 20$ & 8.7199E-08 & 9.10 & $1.4662 \mathrm{E}-07$ & 9.50 \\
\hline & $40 \times 40$ & $1.5470 \mathrm{E}-10$ & 9.14 & $2.5558 \mathrm{E}-10$ & 9.16 \\
\hline & $80 \times 80$ & $1.3857 \mathrm{E}-12$ & 6.80 & $1.9387 \mathrm{E}-12$ & 7.04 \\
\hline
\end{tabular}

\section{IV.d) Dispersion and Dissipation Accuracy of WENO Schemes}

WENO schemes are increasingly used for turbulence studies. In such studies, practitioners are very interested in knowing the dispersion error and dissipation characteristics of their numerical methods. The modified wavenumber approach, popularized by Lele [21], gives us a measure of the dispersion error as well as the dissipation inherent in the scheme when a first derivative is taken. Since hyperbolic PDEs are based on taking a first derivative of the flux, this is a useful diagnostic. The real part of the modified wave number for the first derivative gives us a good measure of the dispersion error of the scheme. Since all our WENO schemes are upwind schemes, they will indeed have some dissipation. However, one would like this dissipation to be as small as possible over a large range of intermediate wave numbers in a higher order scheme. 
The previous Sub-sections have shown that for smooth enough flow the WENOAO schemes achieve their design accuracies. Consequently, we use the highest order stencil in the dispersion and dissipation analysis that is described in the next four paragraphs.

Fig. 1a shows the real part of the modified wave number for the first derivative as a function of wave number. The solid straight line shows the modified wave number for the first derivative under exact differentiation. No stable scheme will ever produce that result. The solid curve in Fig. 1a shows the real part of the modified wave number for the fourth order Padé scheme. This Padé scheme requires the solution of a tri-diagonal system along each row of the computation, which impedes massively parallel computation. We see that the fourth order Padé scheme approximates the solid straight line for a large range of wave numbers. This is inevitable, considering that the Padé scheme is designed to minimize the dispersion error. The curve with short dashes in Fig. 1a shows the real part of the modified wave number for the fourth order central finite difference scheme; we note that its dispersion error is indeed quite significant. The WENO-AO $(5,3)$ has a maximum fifth order of accuracy and the real part of its modified wave number is shown in Fig. 1a by the long dashed curve. We see that its dispersion properties compare favorably to the fourth order Padé scheme. Besides, WENO-AO $(5,3)$ is an explicit scheme and does not require the solution of a tridiagonal system; this is a significant advantage for parallel computations. The solid curve in Fig. 2 shows the imaginary part of the modified wave number for the first derivative as a function of wave number. This is a measure of the dissipation of the upwind scheme. We see that WENO$\mathrm{AO}(5,3)$ results in some dissipation over a substantial range of intermediate wave numbers.

Fig. 1b shows similar information for the $\operatorname{WENO}-\operatorname{AO}(7,5,3)$ scheme, which is up to seventh order accurate. The description of the solid curve and the short dashed curve is unchanged from Fig. 1a. The long dashed curve shows the real part of the modified wave number for the WENO-AO(7,5,3) scheme. We now see that the $\operatorname{WENO}-\operatorname{AO}(7,5,3)$ 
scheme has dispersion error that compares very favorably with the fourth order Padé scheme and is very superior compared to the fourth order central finite difference scheme. The short dashed curve in Fig. 2 shows the imaginary part of the modified wave number for the first derivative as a function of wave number for the $\operatorname{WENO}-\operatorname{AO}(7,5,3)$ scheme. We see that it provides almost dissipation free propagation of waves over a substantial range of intermediate wave numbers.

Fig. 1c shows the analogous information for the $\operatorname{WENO}-\operatorname{AO}(9,5,3)$ scheme, which is up to ninth order accurate. The solid curve shows the real part of the modified wave number for the sixth order compact scheme from Lele [21]. Lele's sixth order compact scheme requires the inversion of a pentadiagonal matrix for each row of the computation, which impedes massively parallel computation. The short dashed curve shows the real part of the modified wave number for a sixth order central finite difference scheme. The long dashed curve shows the real part of the modified wave number for the WENO-AO $(9,5,3)$ scheme. We see that the dispersion error of the WENO scheme compares quite favorably with the sixth order compact scheme and is superior to the sixth order central finite difference scheme. The long dashed curve in Fig. 2 shows the imaginary part of the modified wave number for the first derivative as a function of wave number for the WENO-AO $(9,5,3)$ scheme. We see that it provides almost dissipation free propagation of waves over a large range of intermediate wave numbers.

For the applications that we are interested in, it is not useful to design an eleventh order accurate WENO-AO scheme. While we haven't implemented a WENO-AO(11,5,3) scheme, it is possible to show the reader some of its dispersion and dissipation properties. Fig. 1d shows the dispersion properties of the $\operatorname{WENO-AO}(11,5,3)$ scheme. The description of the solid curve and the short dashed curve is unchanged from Fig. 1c. The long dashed curve shows the real part of the modified wave number for the WENO$\mathrm{AO}(11,5,3)$ scheme. We see that it compares very favorably with a sixth order accurate compact scheme. The dotted curve in Fig. 2 shows the imaginary part of the modified wave number for the first derivative as a function of wave number for the WENO- 
$\mathrm{AO}(11,5,3)$ scheme. We see that it provides truly dissipation-free propagation of waves over a very large range of intermediate wave numbers.

\section{V) Test Problems}

Here we present several stringent test problems that are drawn from the literature. The first four test problems are one-dimensional and are run with a CFL 0f 0.6; the next three test problems are two-dimensional and are run with a CFL of 0.3 . The third order accurate SSP-RK3 timestepping scheme from Shu and Osher [29] was used for all these tests. For all the problems where a hyperbolic system is solved, local characteristic limiting was used with an LLF-based Riemann solver to avoid spurious oscillations. For multidimensional test problems, the reconstruction was carried out in a dimension-bydimension fashion. The schemes were all run without any steepening process so that the native capability of the schemes is shown. Furthermore, we did not use any monotonicity preservation methods because all the schemes are eventually stabilized by their nonlinear hybridization with the $r=3 \mathrm{CWENO}$ scheme.

\section{V.a) The Scalar Advection Test Problem}

In various fields, such as astrophysics and space physics, complicated waveforms have to be advected on the mesh without any distortion. Lower order schemes are prone to excessive dispersion and dissipation and also to the formation of top hat profiles, when they propagate flow features over large distances. Higher order schemes are very valuable because they can propagate waveforms over large distances on a computational mesh with minimal distortion. This test problem, originally designed by Jiang and Shu [19], is designed to show the capability of higher order schemes. The problem has several shapes that are difficult to advect with fidelity. The shapes consist of : 1) a combination of Gaussians, 2) a square wave, 3) a sharply peaked triangle and 4) a half ellipse arranged initially from left to right. It is a stringent test problem because it has a combination of

functions that are not smooth and functions that are smooth but sharply peaked. The 
Gaussians differ from the triangle in that the Gaussians' profile actually has an inflection in the second derivative. A good numerical method that can advect information with a high level of fidelity must be able to preserve the specific features of the problem that we have catalogued above. For a full and detailed description of this well-known problem, we refer the reader back to Jiang and Shu [19] or Balsara and Shu [1].

The problem was initialized on a mesh of 400 zones. It was run for a simulation time of 20 which corresponds to ten traversals around the mesh. In doing so, the flow features were advected over 4000 mesh points. The RF-Riemann solver was used in order to produce a crisper solution. Figs. $3 \mathrm{a}, 3 \mathrm{~b}$ and $3 \mathrm{c}$ show the results of the advection test problem for the WENO-ZQ, WENO-AO(5,3) and WENO-AO(7,5,3) schemes respectively. We see that schemes with increasing order of accuracy show an improvement in their advection properties. The schemes were all run without any steepening process so that the native capability of the schemes is shown. We also ran the WENO-AO $(9,5,3)$ scheme for this test problem. However, for a 400 zone treatment of this problem, the result from the WENO-AO $(9,5,3)$ scheme shows no improvement over the WENO-AO(7,5,3) scheme, consequently, the result from the $\operatorname{WENO}-\operatorname{AO}(9,5,3)$ scheme is not shown.

The WENO-ZQ result in Fig. 3a deserves special attention. When we ran it using eqns. (2.19) and (2.20) of the paper by Zhu and Qiu [37], along with their suggested value of $\gamma_{1}=0.98$, we saw overshoots and undershoots. We then realized that the presence of the square in their eqn. (2.19) made their evaluation of the non-linear weights solution-dependent. Fig. 3a was obtained after we removed the square in their eqn. (2.19). The resulting Fig. 3a shows rather excessive flattening in the combination of Gaussians and the sharply peaked triangle. It also shows some slight flattening in the profile for the half ellipse. This is inevitable considering that the Van Albada limiter is non-linearly hybridized in the WENO-ZQ scheme. We should also mention that removing the square in eqn. (2.19) of Zhu and Qiu [37] had a significantly negative effect on the accuracy. As a result, Tables I, II and III show the results from WENO-ZQ without any modification by us. We made this choice so that WENO-ZQ may be portrayed in the best light. 


\section{V.b) The Lax Test Problem}

The Lax shock tube test problem is very well known in fluid dynamics, so we do not repeat its description here. The problem is initialized on a 200 zone domain $x \in[-0.5,0.5]$ and is run to a time of 0.16 by which time a right-going shock, a left-going rarefaction fan and an intermediate contact discontinuity establish themselves. Figs. 4a and $4 \mathrm{~b}$ show the density profile from the Lax test problem for the $\operatorname{WENO}-\operatorname{AO}(5,3)$ and WENO-AO $(9,5,3)$ schemes respectively. The solid line in those figures shows the analytical solution to the Lax Riemann problem. The contact discontinuity is very wellpreserved and there are no overshoots or undershoots.

\section{V.c) The Interacting Blast Wave Test Problem}

We have run the interacting blast wave problem from Woodward and Colella [36] using exactly the same parameters used by those authors. Figs. 5 shows the density profile from a 400 zone simulation of the interacting blast problem with open circles for the WENO-AO $(5,3)$ scheme. The solid line shows the converged density obtained from a 1000 zone simulation. We see that all the density features are captured very well and the 400 zone simulation is very close to the converged density profile from the 1000 zone simulation.

\section{V.d) The Shock-Density Wave Interaction Test Problem}

Higher order schemes should show their advantages when the solution contains an interaction between shocks and complex smooth flow features. Shu and Osher [30] presented a problem where a Mach 3 shock wave interacts with a density disturbance and generates a flow field that has a combination of smooth structures and discontinuities. It is a good model for the kinds of interactions that occur in simulations of compressible turbulence. It represents the amplification of entropy fluctuations as they pass through a strong shock. We use the exact same parameters as Shu and Osher [30] and we run the 
problem to a final time of 1.8 units. A reference solution was generated on a 2000 zone mesh with a fifth order WENO scheme. Fig. 6 shows the final density for the shockdensity wave interaction on a 400 zone mesh shown as circles, with the reference solution from the 2000 zone mesh shown as a solid line. Fig. 6a shows the full solution from a WENO-AO $(5,3)$ scheme while Fig. $6 \mathrm{~b}$ shows a zoom-in of the interaction region spanning $[-0.25,3.0]$. Figs. $6 \mathrm{c}$ and $6 \mathrm{~d}$ show similar zoom-ins from simulations with WENO-ZQ and WENO-AO $(9,5,3)$ schemes. We see that the higher order schemes do a slightly better job of coming closer to the converged solution. For the WENO-ZQ scheme we used the settings from eqns. (2.19) and (2.20) of the original paper.

\section{V.e) The Double Mach Reflection Test Problem}

This problem was originally suggested by Woodward and Colella [36] and we fully implement their set up. The problem consists of a strong Mach 10 shock that is initially incident on a reflecting wedge that makes an angle of $60^{\circ}$ with the plane of the shock. The problem was run on a $1600 \times 400$ zone mesh to a final time of 0.2 units. A contact discontinuity emanated from the roll up of the stronger of the two Mach stems. This discontinuity is known to go Kelvin-Helmholtz unstable when sufficient resolution is provided to the simulation. The Kelvin-Helmholtz develops rapidly only if the simulation code is sufficiently non-dissipative. Fig. 7 shows the final density for the double Mach reflection problem. Fig. 7a shows the full domain from a simulation using the WENO-AO(7,5,3) scheme. Fig. $7 \mathrm{~b}$ shows a zoom-in of the Mach stem roll-up when

the WENO-AO $(7,5,3)$ scheme is used. We see that we obtain a very well-resolved roll-up of the Mach stem.

\section{V.f) The Forward Facing Step Test Problem}

This test problem was also proposed by Woodward and Colella [36] and consists of a Mach 3 wind tunnel with a forward-facing step. As the bow shock reflects off the step and then the top wall of the wind tunnel, it establishes a triple-point structure. A vortex sheet emanates from the triple point. We have fully implemented the set-up from 
Woodward and Colella [36]. While the problem was run to a final time of 4 units on a $600 \times 200$ zone mesh, a very interesting vortex sheet roll-up manifests itself at a time of 3 units. The results of the flow density are, therefore, shown at a time of 3 units. Fig. 8a shows the density from the forward facing step simulation at a time of 3 units when the WENO-AO $(7,5,3)$ scheme was used. Fig. 8b shows the same for the WENO-ZQ scheme. The vortex sheet roll-up is captured very crisply by this higher order scheme. At this resolution all the WENO-AO schemes do a very good job of capturing the vortex sheet roll-up, so we only show one of them.

\section{V.g) The Shock-Vortex Interaction Test Problem}

This test problem studies the interaction of a vortex with a shock and was first suggested by Pao and Salas [26]. We use the problem specification from Balsara and Shu [1] which displays the situation where the vortex flows towards the right-upper corner of the mesh and the shock normal makes an angle of $45^{\circ}$ with respect to the $\mathrm{x}$-axis. Since the problem is described in great detail in Balsara and Shu [1], we do not repeat the description here. The problem was run on a $150 \times 150$ zone mesh on a domain spanning $[0,1.5] \times[0,1.5]$. The problem was run to a final simulation time of 0.8 , by which time the vortex has passed through the shock. Figs. $9 \mathrm{a}$ and $9 \mathrm{~b}$ show the density at a final time of 0.8 for the shock-vortex interaction problem for the WENO-AO $(5,3)$ and WENO$\mathrm{AO}(9,5,3)$ schemes. Both simulations yield good results, showing that the recursive formulation presented here extends stably to higher orders. We see that the higher order scheme has retained a little more post-shock structure in the vortex.

\section{V.h) Timing Tests for Multidimensional Problems}

The WENO-AO algorithms make a small reduction in the number of stencil evaluations. However, there is also one rather very large stencil to account for. It is, therefore, interesting to ask how the time to solution for the WENO-AO algorithms compares with the time to solution of the WENO-JS algorithm at fifth order and the MPWENO algorithms at seventh and ninth order. Most readers would be interested in the 
performance for a range of multidimensional test problems, which is why we focus on the Double Mach Reflection (DMR) test problem, the Forward Facing Step (FFStep) test problem and the Shock-Vortex Interaction (SVI) test problem from the previous three Sub-sections. The problems were run at their stated resolutions. We also use the same SSP-RK3 timestepping strategy for all these test problems. To ensure a fair comparison, the smoothness indicators from this paper were used for all the WENO variants.

The results for the time to solution are shown in Table IV. In all instances, we have normalized the time to solution for the fifth order WENO-JS algorithm to unity. Therefore, a number that is less than unity indicates a relative speed-up compared to WENO-JS. A number that is larger than unity indicates a slower speed than WENO-JS. As expected, the algorithms with seventh and ninth order accuracy cost more than WENO-JS. It is interesting to note though that WENO-AO $(5,3)$ costs less than fifth order WENO-JS. Likewise, WENO-AO(7,3) costs less than MPWENO at seventh order; in fact, it costs almost the same as fifth order WENO-JS! Similarly, WENO-AO(9,3) costs less than MPWENO at ninth order; in fact it costs only $\sim 20 \%$ more than the fifth order WENO-JS! Please also note that the test problems in Table IV have been run on different sized meshes and the numbers from larger meshes (like the mesh from the double Mach reflection problem) are more reliable.

There is one very startling result in Table IV. It is that the seventh and ninth order WENO variants do not cost much more than their fifth order counterparts! This very desirable result is certainly a consequence of the very concise smoothness indicators that were invented in this paper. If we had used the smoothness indicators from Balsara \& Shu [1] the time to solution for the seventh and ninth order MPWENO schemes would certainly have been greater. The results show that the concise evaluation of the smoothness indicators makes the code so cache friendly that the additional cost of carrying out extra floating point operations at higher orders is very modest. This is the most important insight that we gain from Table IV. 
Table IV shows the relative time to solution for the last three multidimensional test problems with various higher order finite difference WENO algorithms

\begin{tabular}{|l|l|l|l|l|l|l|}
\hline & $\begin{array}{l}\text { WENO-JS } \\
\left(5^{\text {th }} \text { order }\right)\end{array}$ & $\begin{array}{l}\text { WENO- } \\
\mathrm{AO}(5,3)\end{array}$ & $\begin{array}{l}\text { MPWENO } \\
\left(7^{\text {th }} \text { order }\right)\end{array}$ & $\begin{array}{l}\text { WENO- } \\
\mathrm{AO}(7,3)\end{array}$ & $\begin{array}{l}\text { MPWENO } \\
\left(9^{\text {th }} \text { order }\right)\end{array}$ & $\begin{array}{l}\text { WENO- } \\
\mathrm{AO}(9,3)\end{array}$ \\
\hline DMR & 1.0 & 0.87 & 1.23 & 1.06 & 1.48 & 1.22 \\
\hline FFStep & 1.0 & 0.95 & 1.26 & 1.16 & 1.54 & 1.35 \\
\hline SVI & 1.0 & 0.95 & 1.25 & 1.15 & 1.55 & 1.35 \\
\hline
\end{tabular}

\section{VI) Conclusions}

Finite difference WENO schemes have been used extensively in the research literature. They offer high formal accuracy, high phase accuracy and low dissipation. With increasing order of accuracy, these trends are known to improve. In this paper we have presented two major advances in finite difference WENO schemes that are recapitulated in the subsequent paragraphs.

First, we present a one-dimensional reconstruction strategy that uses Legendre polynomials as basis functions. We show that in this formulation the smoothness indicators can be written very compactly as the sum of perfect squares. Since the evaluation of smoothness indicators is a computationally expensive step, finding compact expressions dramatically reduces the cost of the overall scheme. These reductions in computational costs have also been documented. The methods we present should be useful for classical finite difference WENO schemes that can go as high as seventeenth order. The more compact expressions for the smoothness measures also make the code more cache-friendly, and the improvements are quite significant.

Second, we present a new class of finite difference WENO schemes, which we call WENO-AO. The "AO" stands for adaptive order. This adaptive order property is obtained by non-linear hybridization between a higher order centered stencil and a rock- 
stable $r=3$ CWENO scheme. Despite its very good stability properties, the CWENO scheme does not clip physical extrema. Several very successful WENO-AO schemes are presented. Thus we arrive at a WENO-AO $(5,3)$ scheme that is at best fifth order accurate by virtue of its centered stencil with five zones and at worst third order accurate by virtue of being non-linearly hybridized with an $r=3$ CWENO scheme. The process can be extended to arrive at a WENO-AO(7,3) scheme that is at best seventh order accurate by virtue of its centered stencil with seven zones and at worst third order accurate. We then recursively combine the above two schemes to arrive at a $\operatorname{WENO}-\mathrm{AO}(7,5,3)$ scheme which can achieve seventh order accuracy when that is possible; graciously drop down to fifth order accuracy when that is the best one can do; and also operate stably with an $r=3$ CWENO scheme when that is the only thing that one can do. Schemes with ninth order of accuracy are also presented. The resulting schemes do not seem to need any monotonicity preserving step. They also seem to capture discontinuities very crisply without needing any steepening step.

Accuracy analysis is presented to show that the methods meet their design accuracies. The dispersion and dissipation properties of these schemes is also documented using a modified wave number approach. It is shown that WENO-AO schemes with increasing order show progressively decreasing dispersion and dissipation, making them very attractive performers for fluid dynamical turbulence studies. Several stringent test problems are also presented to show that the methods work very well.

\section{Acknowledgements}

DSB acknowledges support via NSF grants NSF-ACI-1307369, NSF-DMS1361197 and NSF-ACI-1533850. DSB also acknowledges support via NASA grant NASA-NNX 12A088G. CWS acknowledges support via ARO grant W911NF-15-1-0226 and NSF grant DMS-1418750. Several simulations were performed on a cluster at UND that is run by the Center for Research Computing. Computer support on NSF's XSEDE computing resources is also acknowledged. 


\section{References}

[1] D. S. Balsara and C.-W. Shu, Monotonicity Preserving Weighted Non-oscillatory schemes with increasingly High Order of Accuracy, Journal of Computational Physics, $160(2000) 405-452$

[2] D. S. Balsara, T. Rumpf, M. Dumbser \& C.-D. Munz, Efficient, high-accuracy ADER-WENO schemes for hydrodynamics and divergence-free magnetohydrodynamics, Journal Computational Physics, 228 (2009) 2480

[3] Borges, R., Carmona, M., Costa, B., Don, W.S., An improved weighted essentially non-oscillatory scheme for hyperbolic conservation laws, Journal of Computational Physics, 227 (6) (2008) 3101-3211

[4] M. Castro, B. Costa, W.S. Don, High order weighted essentially non-oscillatory WENO-Z schemes for hyperbolic conservation laws, J. Comput. Phys. 230 (2011) 17661792

[5] S. Clain, S. Diot and R. Loubère, A high-order finite volume method for systems of conservation laws-Multi-dimensional Optimal Order Detection (MOOD), Journal of Computational Physics 230 (2011) 4028-4050

[6] I. Cravero and M. Semplice, On the Accuracy of WENO and CWENO Reconstructions of Third Order on Nonuniform Meshes, Journal of Scientific Computing, Volume 67, Issue 3, 1 June 2016, Pages 1219-1246

[7] X. Deng and H. Zhang, Developing high order weighted compact nonlinear schemes, Journal of Computational Physics, 203 (2005) 22-44 
[8] S. Diot, S. Clain and R. Loubère, Improved detection criteria for the Multidimensional Optimal Order Detection (MOOD) on unstructured meshes with very highorder polynomials, Computers \& Fluids 64 (2012) 43-63

[9] S. Diot, R. Loubère and S. Clain, The Multidimensional Optimal Order Detection method in the three-dimensional case: very high-order finite volume method for hyperbolic systems, Int. J. Numer. Meth. Fluids 73 (2013) 362-392

[10] M., Dumbser, M., Käser, Arbitary high order non-oscillatory finite volume schemes on unstructured meshes for linear hyperbolic systems, Journal of Computational Physics, 221 (2007) 693-723

[11] M. Dumbser, O. Zanotti, R. Loubere, S. Diot, A posteriori subcell limiting of the discontinuous Galerkin finite element method for hyperbolic conservation laws, J. Comp. Phys., Vol. 278 (2014) 47-75

[12] O. Friedrichs, Weighted essentially non-oscillatory schemes for the interpolation of mean values on unstructured grids, Journal of Computational Physics, 144 (1998) 194212

[13] G.A. Gerolymos, D. Sénéchal and I. Vallet, Very high order WENO schemes, Journal of Computational Physics, 228 (2009) 8481-8524

[14] A. Harten, B. Engquist, S.Osher and S. Chakravarthy, Uniformly high order essentially non-oscillatory schemes III, Journal of Computational Physics, 71 231-303

[15] A.K. Henrick, T.D. Aslam and J.M. Powers, Mapped weighted essentially nonoscillatoriy schemes: Achieving optimal order near critical points, Journal of Computational Physics 207 (2006) 542-567 
[16] C. Hu and C.-W. Shu, Weighted essentially non-oscillatory schemes on triangular meshes, J. Comput. Phys., 150 (1999), 97-127

[17] M., Käser and A. Iske, ADER schemes on adaptive triangular meshes for scalar conservation laws, Journal of Computational Physics 205 (2005) 486-508

[18] Hu, X.Y., Wang, Q. and Adams, N.A., An adaptive central-upwind weighted essentially non-oscillatory scheme, Journal of Computational Physics, 229 (2010) 89528965

[19] G.-S. Jiang and C.-W. Shu, Efficient implementation of weighted ENO schemes, Journal of Computational Physics 126 (1996) 202-228

[20] E. Johnsen et al., Assessment of high-resolution methods for numerical simulations of compressible turbulence with shock waves, Journal of Computational Physics, 229 (2010) 1213-1237

[21] S. Lele, Compact finite difference schemes with spectral-like resolution, Journal of Computational Physics, 103 (1992), 16-42

[22] D. Levy, G. Puppo and G. Russo, Compact central WENO schemes for multidimensional conservation laws, SIAM J. Sci. Comput. 22 (2000) 656-672

[23] X.-D. Liu, S. Osher and T. Chan, Weighted essentially non-oscillatory schemes, Journal of Computational Physics 115 (1994) 200-212

[24] Y. Liu and Y.T. Zhang, A robust reconstruction for unstructured WENO schemes, Journal of Scientific Computing, 54 (2013), 603-621 
[25] M.P. Martin, E.M. Taylor, M. Wu and V.G. Weirs, A bandwidth-optimized WENO scheme for the effective direct numerical simulation of compressive turbulence, 220 (2006) 270-289

[26] S.P. Pao and M.D. Salas, A numerical study of two-dimensional shock-vortex interaction, AIAA Paper 81-1205 (1981) $14^{\text {th }}$ Fluid and Plasma Dynamics Conference

[27] S. Pirozzoli, Conservative hybrid compact-WENO schemes for shock-turbulence interaction, J. Comput. Phys., 178 (2002), 81-117

[28] M. Semplice, A. Coco, G. Russo. Adaptive Mesh Refinement for Hyperbolic Systems Based on Third-Order Compact WENO Reconstruction, Journal of Scientific Computing, Volume 66, Issue 2, 1 February 2016, Pages 692-724

[29] C.-W. Shu and S. J. Osher, Efficient implementation of essentially non-oscillatory shock capturing schemes, Journal of Computational Physics, 77 (1988) 439-471

[30] C.-W. Shu and S. J. Osher, Efficient implementation of essentially non-oscillatory shock capturing schemes II, Journal of Computational Physics, 83 (1989) 32-78

[31] Shu, C.-W., High order weighted essentially non-oscillatory schemes for convection dominated problems, SIAM Review, 51 (2009) 82-126

[32] Y.Q. Shen and G.W. Yang, Hybrid finite compact-WENO schemes for shock calculation, Interational Journal for Numerical Methods in Fluids, 53 (2007), 531-560

[33] Spiteri, R.J. and Ruuth, S.J., A new class of optimal high-order strong-stabilitypreserving time-stepping schemes, SIAM Journal of Numerical Analysis, 40 (2002), pp. $469-491$ 
[34] Spiteri, R.J. and Ruuth, S.J., Non-linear evolution using optimal fourth-order strongstability-preserving Runge-Kutta methods, Mathematics and Computers in Simulation 62 (2003) 125-135

[35] C.K. Tam and J.C. Webb, Dispersion-relation-preserving finite difference schemes for computational acoustics, Journal of Computational Physics, 107 (1993) 262-281

[36] P. Woodward and P. Colella, The numerical simulation of two-dimensional fluid flow with strong shocks, Journal of Computational Physics 54 (1984), 115-173

[37] J. Zhu and J. Qiu, A new fifth order finite difference WENO scheme for solving hyperbolic conservation laws, accepted, Journal of Computational Physics (2016)

\section{Figure Captions}

Figs. $1 a, 1 b, 1 c$ and $1 d$ show the real part of the modified wavenumbers for the $5^{\text {th }}, 7^{\text {th }}, 9^{\text {th }}$ and $11^{\text {th }}$ order WENO schemes respectively. Curves with big dashes show WENO-AO schemes. Figs. $1 a$ and $1 b$ also show the standard fourth order Padé scheme (solid curve) and the fourth order central finite difference scheme (small dashed curve). Figs. 1c and $1 d$ also show the show the sixth order compact scheme (solid curve) and the sixth order central finite difference scheme (small dashed curve). The solid straight line shows exact differentiation.

Fig. 2 shows the imaginary part of the modified wave number as a function of wave number. This provides a measure of the dissipation for the WENO-AO schemes. Solid, small dash, large dash and dotted curves show the results for $5^{\text {th }}, 7^{\text {th }}, 9^{\text {th }}$ and $11^{\text {th }}$ order WENO-AO schemes respectively.

Figs. $3 a, 3 b$ and $3 c$ show the results of the advection test problem for the WENO-ZQ, WENO-AO(5,3) and WENO-AO(7,5,3) schemes respectively. The advected shapes consist 
of : 1) a combination of Gaussians, 2) a square wave, 3) a sharply peaked triangle and 4) a half ellipse arranged initially from left to right. The flow features were advected over 4000 mesh points.

Figs. $4 a$ and $4 b$ show the density profile from the Lax test problem for the WENO$A O(5,3)$, and WENO-AO $(9,5,3)$ schemes respectively.

Figs. 5 show the density profile from a 400 zone simulation of the interacting blast problem with open circles for the WENO-AO(5,3) scheme. The solid line shows the converged density obtained from a 1000 zone simulation.

Fig. 6 shows the final density for the shock-density wave interaction on a 400 zone mesh shown as circles, with the reference solution from the 2000 zone mesh shown as a solid line. Fig. $6 a$ shows the full solution from a WENO-AO(5,3) scheme while Fig. $6 b$ shows a zoom-in of the interaction region spanning [-0.25,3.0]. Figs. 6c and 6d show similar zoom-ins from simulations with WENO-ZQ and WENO-AO(9,5,3) schemes.

Fig. $7 a$ shows the full domain from a simulation using the WENO-AO(7,5,3) scheme. Fig. $7 b$ shows a zoom-in of the Mach stem roll-up when the WENO-AO(7,5,3) scheme is used. We see that we obtain a very well-resolved roll-up of the Mach stem.

Fig. 8a shows the density from the forward facing step simulation at a time of 3 units when the WENO-AO(7,5,3) scheme was used. Fig. $8 b$ shows the same for the WENO-ZQ scheme. The vortex sheet roll-up is captured very crisply by this higher order scheme.

Figs. $9 a$ and $9 b$ show the density at a final time of 0.8 for the shock-vortex interaction problem for the WENO-AO(5,3) and WENO-AO(9,5,3) schemes. Both simulations yield good results, showing that the recursive formulation presented here extends stably to higher orders. 


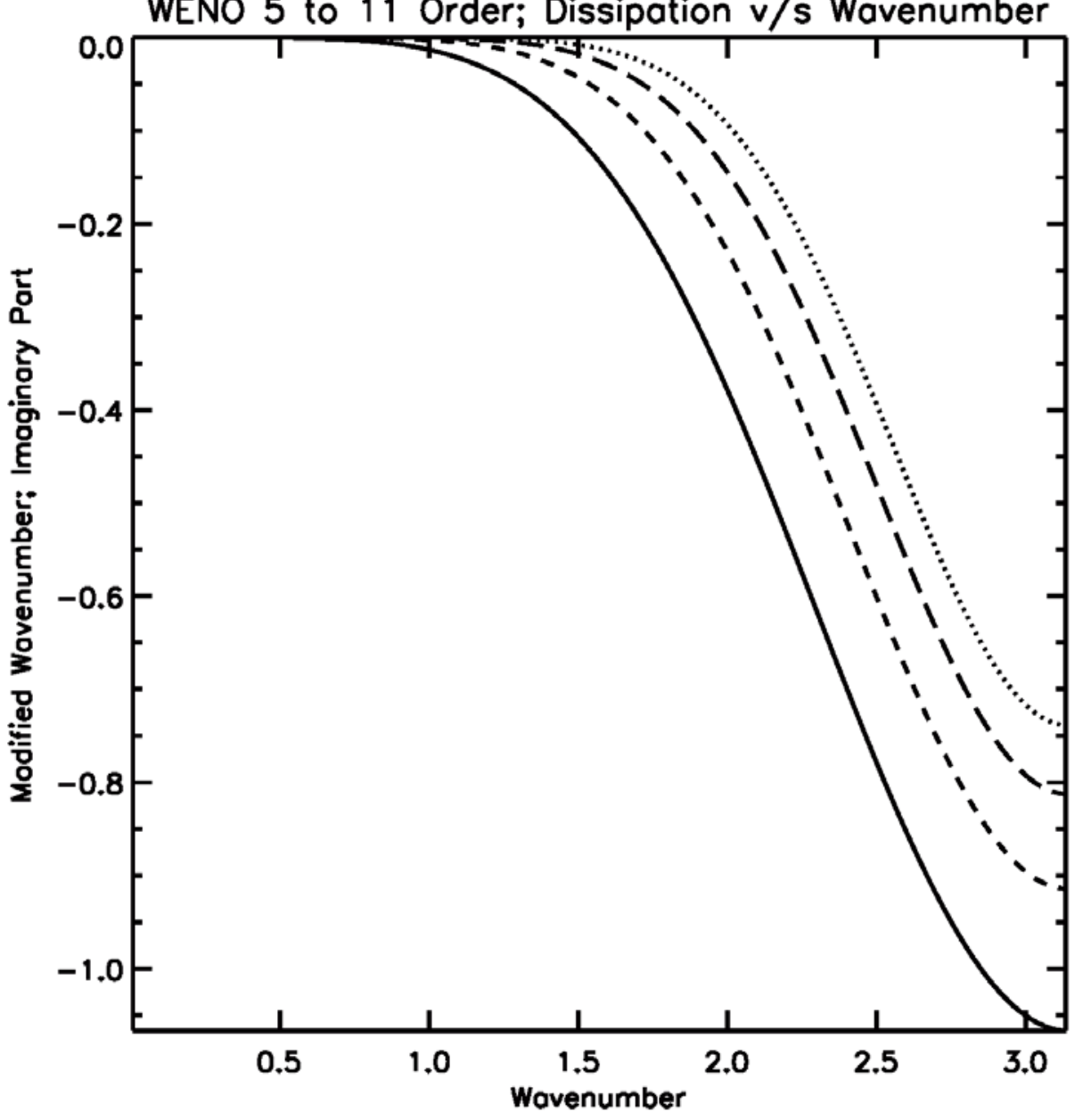

Fig. 2 shows the imaginary part of the modified wave number as a function of wave number. This provides a measure of the dissipation for the WENO-AO schemes. Solid, small dash, large dash and dotted curves show the results for $5^{\text {th }}, 7^{\text {th }}, 9^{\text {th }}$ and $11^{\text {th }}$ order WENO-AO schemes respectively. 

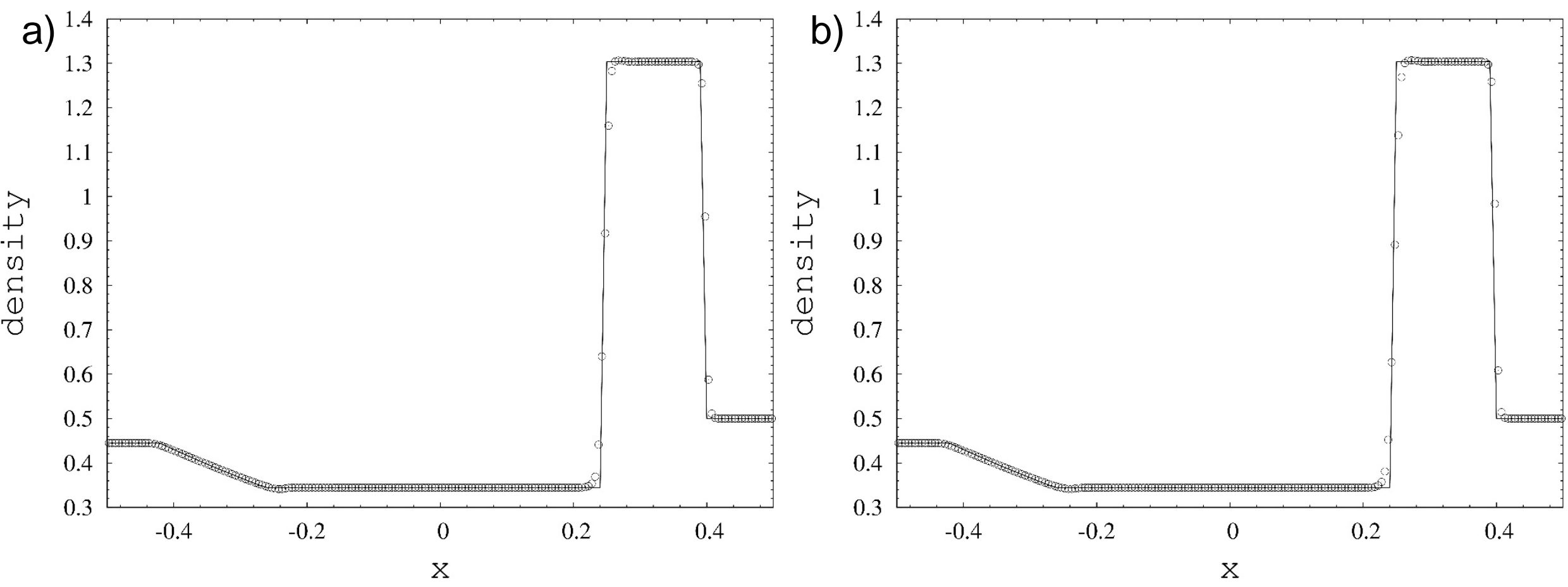

Figs. $4 a$ and $4 b$ show the density profile from the Lax test problem for the WENO-AO(5,3), and WENO-AO(9,5,3) schemes respectively. 


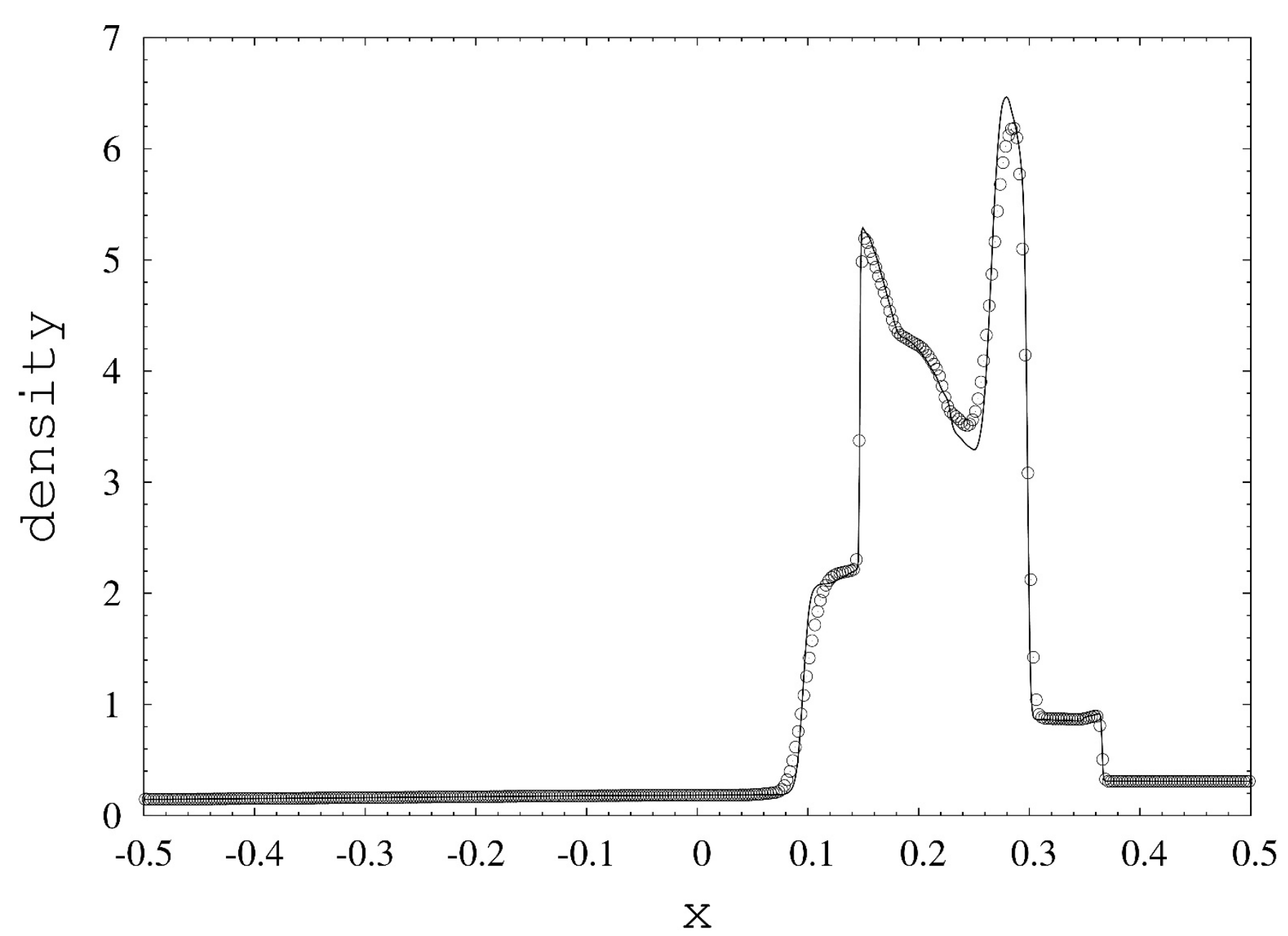

Figs. 5 show the density profile from a 400 zone simulation of the interacting blast problem with open circles for the WENO-AO(5,3) scheme. The solid line shows the converged density obtained from a 1000 zone simulation. 
a)

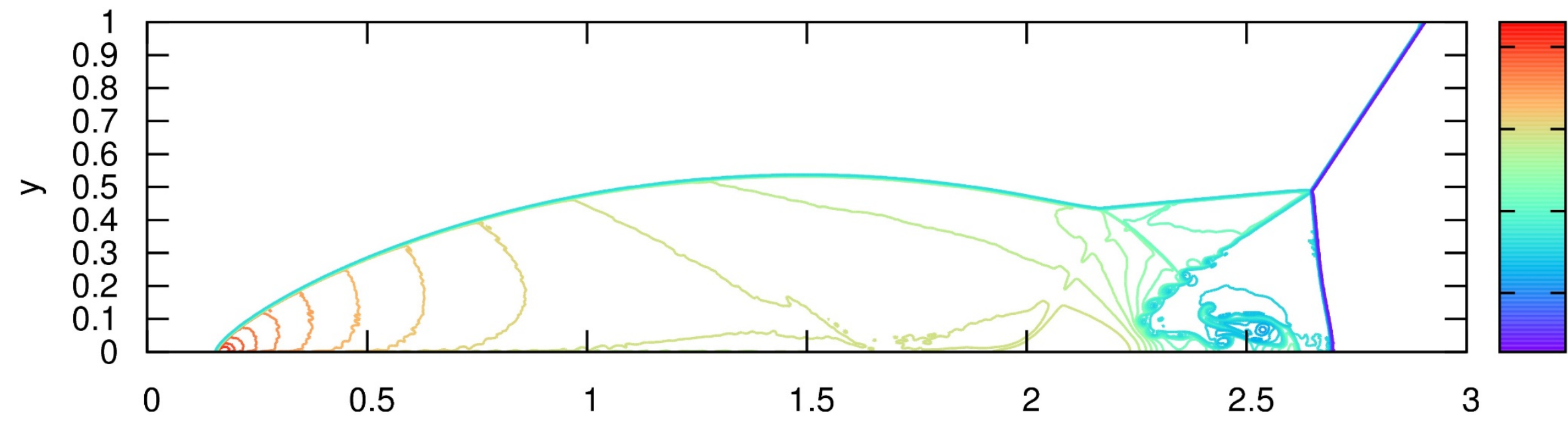

b)

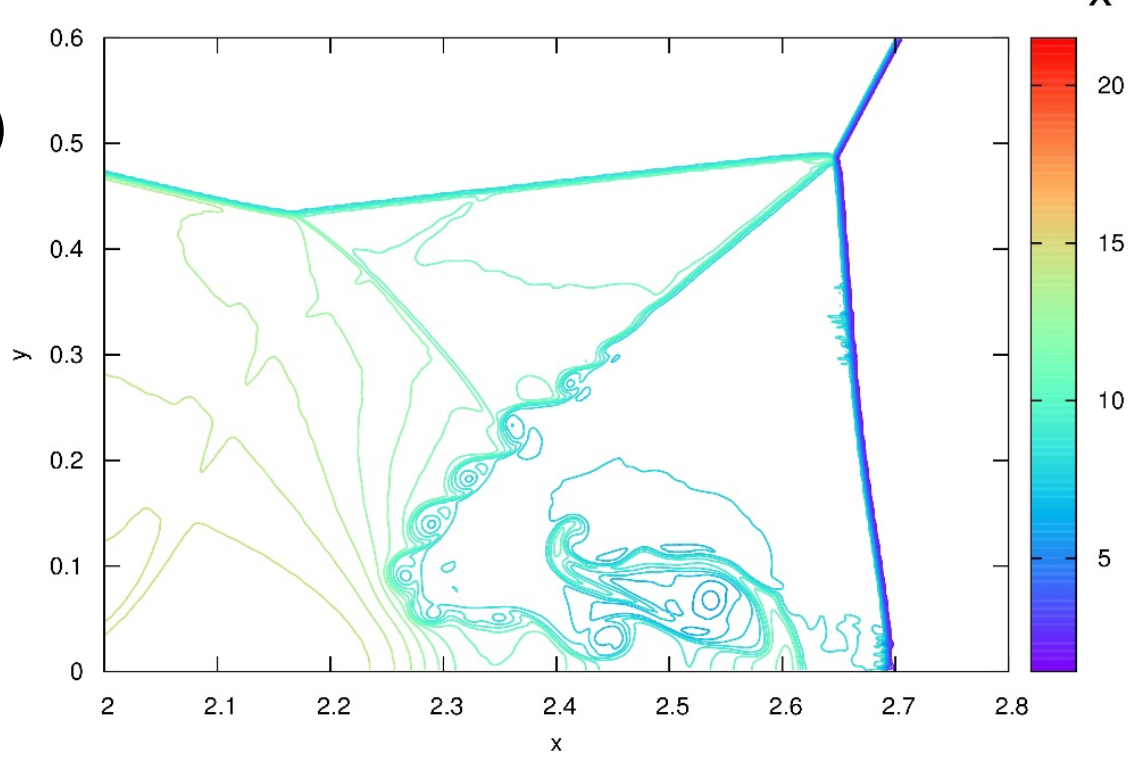

Fig. 7a shows the full domain from a simulation using the WENO-AO(7,5,3) scheme. Fig. $7 \mathrm{~b}$ shows a zoom-in of the Mach stem roll-up when the WENO-AO(7,5,3) scheme is used. We see that we obtain a very well-resolved roll-up of the Mach stem. 

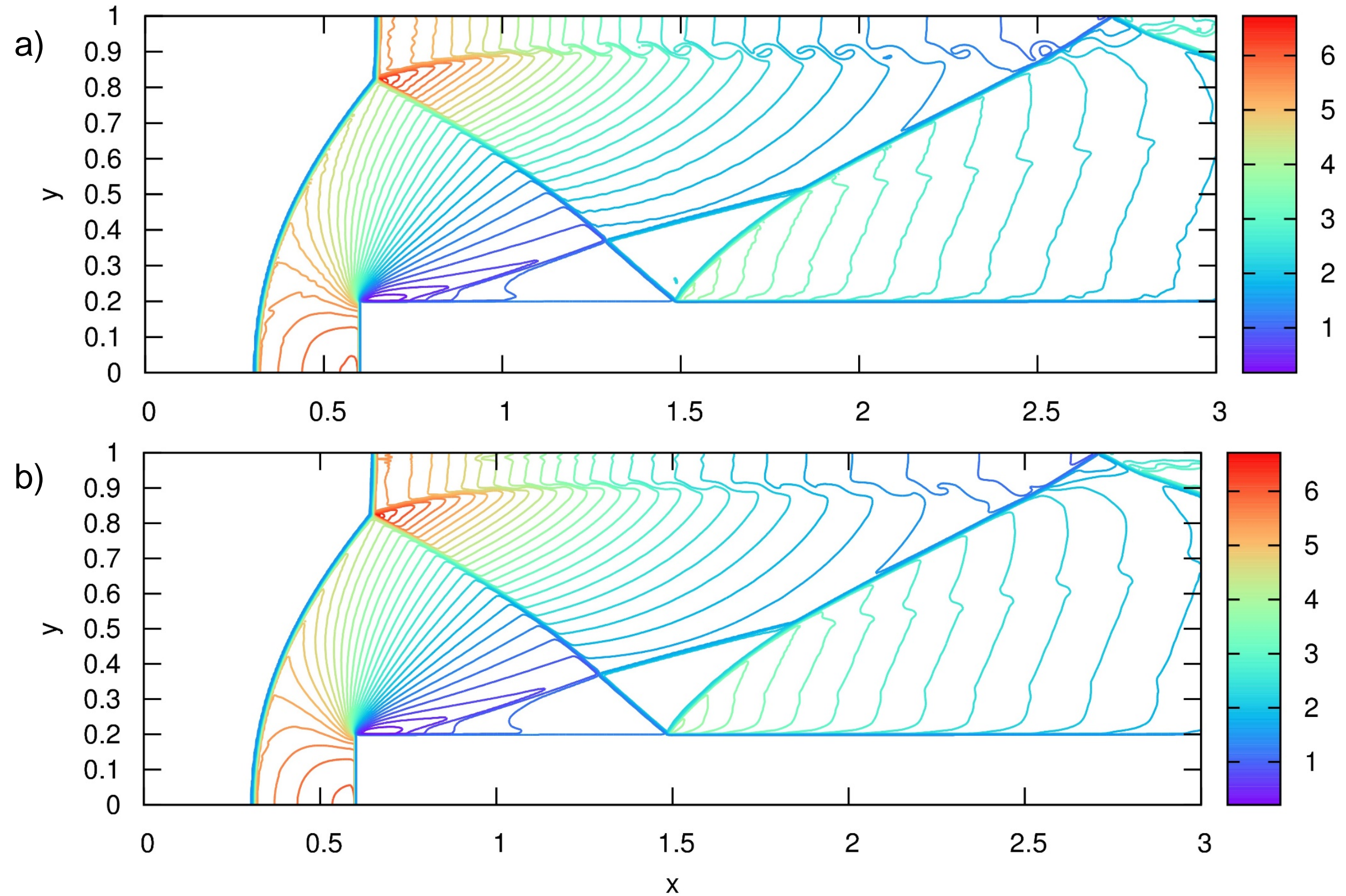

Fig. 8a shows the density from the forward facing step simulation at a time of 3 units when the WENO-AO(7,5,3) scheme was used. Fig. $8 b$ shows the same for the WENO-ZQ scheme. The vortex sheet roll-up is captured very crisply by this higher order scheme. 

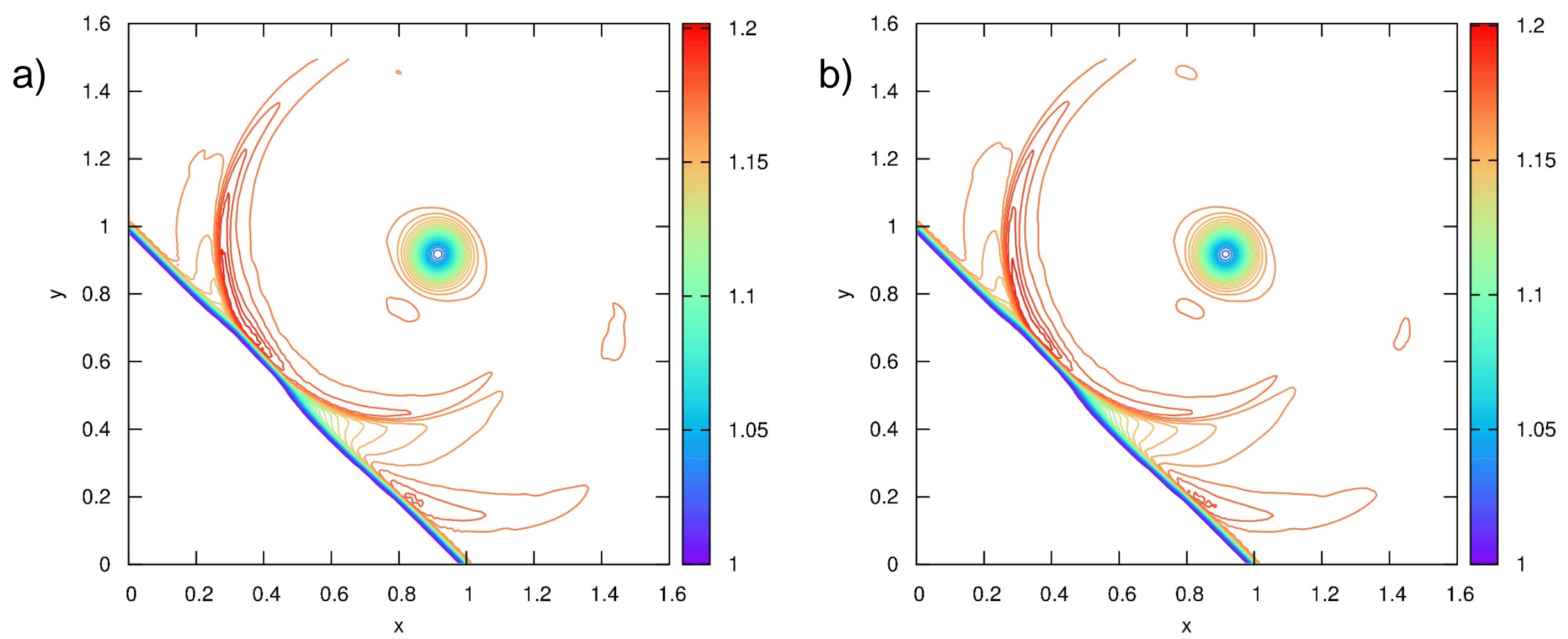

Figs. $9 a$ and $9 b$ show the density at a final time of 0.8 for the shock-vortex interaction problem for the WENO-AO(5,3) and WENO-AO $(9,5,3)$ schemes. Both simulations yield good results, showing that the recursive formulation presented here extends stably to higher orders. 